\title{
Spectral Integration in Primary Auditory Cortex Attributable to Temporally Precise Convergence of Thalamocortical and Intracortical Input
}

\author{
Max F. K. Happel, ${ }^{1,2 \star}$ Marcus Jeschke, ${ }^{1 \star}$ and Frank W. Ohl ${ }^{1,2}$ \\ ${ }^{1}$ Leibniz Institute for Neurobiology, D-39118 Magdeburg, Germany, and 2Institute of Biology, Otto von Guericke University, D-39120 Magdeburg, Germany
}

\begin{abstract}
Primary sensory cortex integrates sensory information from afferent feedforward thalamocortical projection systems and convergent intracortical microcircuits. Both input systems have been demonstrated to provide different aspects of sensory information. Here we have used high-density recordings of laminar current source density (CSD) distributions in primary auditory cortex of Mongolian gerbils in combination with pharmacological silencing of cortical activity and analysis of the residual CSD, to dissociate the feedforward thalamocortical contribution and the intracortical contribution to spectral integration. We found a temporally highly precise integration of both types of inputs when the stimulation frequency was in close spectral neighborhood of the best frequency of the measurement site, in which the overlap between both inputs is maximal. Local intracortical connections provide both directly feedforward excitatory and modulatory input from adjacent cortical sites, which determine how concurrent afferent inputs are integrated. Through separate excitatory horizontal projections, terminating in cortical layers II/III, information about stimulus energy in greater spectral distance is provided even over long cortical distances. These projections effectively broaden spectral tuning width. Based on these data, we suggest a mechanism of spectral integration in primary auditory cortex that is based on temporally precise interactions of afferent thalamocortical inputs and different short- and long-range intracortical networks. The proposed conceptual framework allows integration of different and partly controversial anatomical and physiological models of spectral integration in the literature.
\end{abstract}

\section{Introduction}

Primary sensory cortices often display topographically organized representations of stimulus features derived from the biophysical constraints of sensory transduction processes and subsequent neuronal computations. In the case of auditory cortex, the spectral distribution of acoustic signal energy is such a feature and is dominantly represented in tonotopic maps (for overview, see Schreiner and Winer, 2007). Previous studies have demonstrated several mechanisms by which information from different parts of the signal spectrum is integrated and affects neuronal activity at a given site within the tonotopic map (spectral integration). Both studies of single-unit firing behavior (Ohl and Scheich, 1997; Schreiner et al., 2000; L. M. Miller et al., 2001a,b; Kadia and Wang, 2003) and studies focusing on the subthreshold integration of inputs with cell-attached recording (Wehr and Zador, 2003; Liu et al., 2007) or recording of local field potentials (LFPs) (Kaur et al., 2004, 2005; Metherate et al., 2005) have revealed that thalamic input of spectral information into the cortex is not re-

\footnotetext{
Received Feb. 8, 2010; revised May 17, 2010; accepted June 9, 2010.

This work was supported by Deutsche Forschungsgemeinschaft Grants SFB TRR31 and SFB 779. We thank Kathrin Ohl for technical assistance and Dr. Michael S. Osmanski for valuable comments on a previous version of this manuscript.

*M.F.K.H. and M.J. contributed equally to this work.

Correspondence should be addressed to either Max F. K. Happel or Frank W. Ohl, Leibniz Institute for Neurobiology, Brenneckestrasse 6, D-38118 Magdeburg, Germany, E-mail: mhappel@ifn-magdeburg.de or frank.ohl@ifnmagdeburg.de.

DOI:10.1523/JNEUROSCI.0689-10.2010

Copyright $\odot 2010$ the authors $\quad 0270-6474 / 10 / 3011114-14 \$ 15.00 / 0$
}

layed in a point-to-point manner. In other words, the finegrained suprathreshold representation of frequencies in primary auditory cortex cannot exclusively be inherited from afferent feedforward inputs (L. M. Miller et al., 2001a; Edeline, 2003; Wehr and Zador, 2003; Winer et al., 2005). Different short-range or long-range intracortical connections could potentially also provide spectral input to a given cortical site (Wallace et al., 1991; Budinger et al., 2000b). In fact, several studies have suggested that long-range horizontal pathways can provide (subthreshold) spectral input to adjacent cortical regions not receiving any corresponding thalamic input (Kadia and Wang, 2003; Metherate et al., 2005; Kurt et al., 2008; Moeller et al., 2010). Another recent study showed that intracolumnar circuits that mediate excitation through recurrent loops selectively amplify suprathreshold activation of thalamocortical-recipient neurons, thereby sharpening cortical frequency tuning (Liu et al., 2007).

These data raise the question how the integration of information about the stimulus spectrum from thalamocortical inputs and intracortical inputs is organized in time and space, i.e., across the synaptic populations extending over the dendritic tree of cortical principal neurons. To answer this question, we have combined acoustical stimulation of cortex with pure tones before and after pharmacological silencing of intracortical activity with the analysis of the laminar profiles of tone-evoked current source density (CSD) and of the residual CSD (cf. Harding, 1992).

We found that afferent thalamocortical inputs and convergent intracortical inputs have a discernible spatial organization and are differentially recruited in time. Specifically, when a cor- 
tical site was stimulated with a tone frequency corresponding to its best frequency (BF) or to a spectrally distant frequency (nonBF), we found thalamocortical or intracortical inputs, respectively, to be the dominant generators of the initial cortical activation. For frequencies in the spectral neighborhood of the BF (nearBF), in which both input systems exhibit their greatest overlap, we found a temporally highly precise integration of both inputs to produce a characteristic laminar activation profile.

\section{Materials and Methods}

Experiments were performed on 13 adult male ketamine/xylazineanesthetized Mongolian gerbils (Meriones unguiculatus) (age, 3-16 months; body weight, 80-120 g). All experiments were conducted in accordance with the international National Institutes of Health Guidelines for Animals in Research and with ethical standards for the care and use of animals in research defined by the German Law for the protection of experimental animals. Experiments were approved by an ethics committee of the state Saxony-Anhalt, Germany.

Surgical procedure. Initial anesthesia was induced by inhalation of halothane $(4 \%, 4 \mathrm{~min})$ and was switched to a subcutaneous anesthesia with $45 \%$ ketamine (50 mg/ml; Ratiopharm), 5\% xylazine (Rompun, $2 \%$; BayerVital) and $50 \%$ isotonic sodium chloride solution (154 $\mathrm{mmol} / \mathrm{L}$; Braun). Status of anesthesia was maintained at surgical level by monitoring the hindlimb withdrawal reflex and respiratory rate (Zandieh et al., 2003). Body temperature was kept at $37^{\circ} \mathrm{C}$ by a controlled heating blanket. Before surgery, ear canals and tympanic membranes were controlled using a surgical microscope. After resection of the scalp and the right temporalis muscle, right auditory cortex was exposed by craniotomy $(\sim 3 \times 4 \mathrm{~mm})$. A gold-plated pin (Amphenol) was implanted in the contralateral parietal bone with good contact to the dura mater and was used as a reference. Stereotaxic fixation was achieved by an aluminum bar attached to the frontal bones with dental cement (Paladur; Heraeus Kulzer). Immediately before insertion of the multichannel shaft electrode, a T-shaped cut was made in the dura using a cannula needle.

Manufacturing of multichannel shaft electrodes. All experiments were conducted using custom-made multichannel shaft electrodes made of Teflon-insulated stainless-steel microwires with a diameter of $25 \mu \mathrm{m}$ (California Fine Wire) glued together by baking heat-curable epoxy resin (adapted from Jellema and Weijnen, 1991). Equidistant linear arrangement of individual wires was guaranteed by a matrix of pinholes in a half-dissected Teflon tube (diameter, $200 \mu \mathrm{m}$ ). Teflon tubes were perforated using a stereotactically controlled tungsten electrode. Individual wires were crimped to a Molex connector system (PicoClasp). The linear array of 24-28 electrode contracts (spacing 55-75 $\mu \mathrm{m}$ ) spanned $\sim 1650$ $\mu \mathrm{m}$. Impedances were checked for each channel separately and found to be in the range of $200-600 \mathrm{k} \Omega$.

Electrophysiological recordings. Recordings were performed in an acoustically and electrically shielded recording chamber. CSD profiles were calculated offline from the depth profiles of the LFP measured with our custom-shaft multielectrodes (see below). Neuronal population activity was recorded using a multichannel recording system (Multichannel Acquisition Processor; Plexon). Potentials were preamplified $(500 \times)$, bandpass filtered between 3 and $170 \mathrm{~Hz}$ ( $3 \mathrm{~dB}$ cutoff frequency) and digitized at $2 \mathrm{kHz}$. Responses were averaged over 100 stimulus repetitions. Recording positions were chosen based on vasculature landmarks and the well established tonotopic organization of primary auditory cortex field A1 (Thomas et al., 1993; Ohl et al., 1997; Schulze et al., 1997). Additionally, we verified the tonotopic gradient at selected locations using spike and LFP recordings obtained with a single tungsten electrode $(0.5-1 \mathrm{M} \Omega)$. The probe was positioned perpendicular to the cortical surface (supplemental Note 2, available at www.jneurosci.org as supplemental material) and rapidly inserted after the dura cut, leaving one to two channels free over the dura. After $\sim 20 \mathrm{~min}$, the cortical tissue distended back until the first channel was directly positioned at the surface. The same set of stimuli was presented before, during, and after epidural application of drugs for cortical silencing.

Acoustical stimulation. Acoustical stimuli were digitally synthesized and controlled using Matlab (MathWorks). We used pseudorandomized series of pure tone frequencies with a logarithmic, i.e., iso-octave, frequency spacing spanning 8 octaves from $125 \mathrm{~Hz}$ to $16 \mathrm{kHz}$. Stimuli were delivered via an attenuator (PA4; Tucker Davis Technologies), an amplifier (STAX SRM-3), and an electrostatic headphone (STAX SR lambda professional) positioned $3 \mathrm{~cm}$ in front of the animal's head. Speaker output was controlled using a $1 / 2$ inch condenser microphone (Brüel \& Kjær). Pure tone frequencies were presented for $200 \mathrm{~ms}$ with $5 \mathrm{~ms}$ sinusoidal rising and falling ramp. In all experiments, we varied intensities in $10 \mathrm{~dB}$ steps from 25 to $85 \mathrm{~dB}$ sound pressure level (SPL). Afterward, we determined the intensity leading to tone-evoked responses $2 \mathrm{SD}$ above baseline. This intensity was declared as the 2 SD threshold intensity. In this study, we analyzed only data with an intensity $20 \mathrm{~dB}$ SPL above this 2 SD threshold intensity (either 65 or $75 \mathrm{~dB}$ SPL). The interstimulus interval was varied between 0.6 and $1 \mathrm{~s}$.

Pharmacological silencing of cortical activity. Intracortical neuronal activity was inhibited using the $\mathrm{GABA}_{\mathrm{A}}$ agonist muscimol hydrobromide (Sigma). A volume of $20 \mu \mathrm{l}$ of muscimol with a concentration of $1 \mu \mathrm{g} / \mu \mathrm{l}$ (8.4 mM) applied topically onto $2.5 \mathrm{~mm}^{2}$ of dura was reported to be an appropriate dosage for effective cortical silencing, including infragranular layers in rat auditory cortex (Talwar et al., 2001). Effective muscimol diffusion is known to be limited to a range of $1-2.5 \mathrm{~mm}$ (Martin and Ghez, 1999; Edeline et al., 2002), and thus muscimol does not reach subcortical structures after topical application. Here, we used different concentrations of $20 \mu \mathrm{l}$ of muscimol alone [ $1 \mu \mathrm{g} / 1 \mu \mathrm{l}(n=5), 0.5 \mu \mathrm{g} / \mu \mathrm{l}$ $(n=3)$, and $0.2 \mu \mathrm{g} / \mu \mathrm{l}(n=2)]$ or concomitantly applied with the $\mathrm{GABA}_{\mathrm{B}}$ receptor agonist (+)-5,5-dimethyl-2-morpholine acetic acid (SCH50911) (6 mM, $20 \mu \mathrm{l} ; n=3$ ) applied on $\sim 12 \mathrm{~mm}^{2}$ of the right hemisphere covering the primary auditory cortex. Cortical silencing using muscimol alone potentially activates $\mathrm{GABA}_{\mathrm{B}}$ receptors $\left(\mathrm{EC}_{50}\right.$ of 25 $\mu \mathrm{M}$ ) (Yamauchi et al., 2000), which would create thalamocortical EPSPs smaller in amplitude attributable to presynaptic inhibition. To avoid this, we adopted an effective method to block the side effects of muscimol on $\mathrm{GABA}_{\mathrm{B}}$ receptors developed by Yamauchi et al. (2000) (see also Liu et al., 2007). Specifically, the $\mathrm{GABA}_{\mathrm{B}}$ receptor agonist SCH50911 (6 mM, 20 $\mu \mathrm{l})$ was applied concomitantly with muscimol $(4 \mathrm{~mm}, 20 \mu \mathrm{l})$ in three additional experiments. It was shown that this mixture still effectively reduced the number of action potentials by $>95 \%$ (Liu et al., 2007). In all cases, we found a strong effect of response inhibition of the laminar LFP and CSD profiles. This blockade was similar in all cases and across different concentrations or drug mixtures. Time until recovery of evoked responses was found to be longer, when muscimol was applied exclusively ( $>10 \mathrm{~h} ; n=5)$, compared with the concomitant application of both drugs (2-4h, $n=3)$. For additional discussion of the adequacy of different silencing techniques, see Results. In a separate study, we used auto-metallographic thallium radiography to demonstrate that activity of cortical neurons in the influenced region was completely blocked, whereas subcortical auditory processing was still active (Goldschmidt et al., 2010).

Current source density analysis. Based on laminar LFP recordings, we calculated the one-dimensional CSD profile from the second spatial derivative of the LFP (Pitts, 1952; Nicholson and Freeman, 1975; Mitzdorf, 1985, 1986; Steinschneider et al., 1992):

$$
-\mathrm{CSD} \approx \frac{\delta^{2} \Phi(z)}{\delta z^{2}}=\frac{\Phi(z+n \Delta z)-2 \Phi(z)+\Phi(z-n \Delta z)}{(n \Delta z)^{2}},
$$

where $\Phi$ is the field potential, $z$ is the spatial coordinate perpendicular to the cortical laminae, $\Delta z$ is the sampling interval $(55-75 \mu \mathrm{m})$, and $n$ is the differentiation grid. Before CSD calculation, LFP profiles were smoothed with a weighted average (Hamming window) of five channels (corresponding to a spatial filter kernel of $300 \mu \mathrm{m}$ ). To retain the upper and lower boundary sites, we used a linear extrapolation method that assumes no additional decay in the second derivative of the field potential. The benefits of a CSD transformation are a reference-free, spatially enhanced representation of the direction, location, and intensity of transmembrane currents underlying the evoked response potential (Nicholson and Freeman, 1975; Mitzdorf, 1985, 1986; Steinschneider et al., 1992). Classically, current sinks are interpreted to indicate depolarizing events such as active excitatory synaptic populations and axonal de- 
polarization. Current sources, in contrast, reflect passive return currents most of the time (Mitzdorf, 1985). Equation 1 takes into account only one dimension, assuming isotropic conductance. Recent findings by Logothetis et al. (2007) strengthen this model of a quasi-ohmic cortical impedance spectrum.

Calculation of peak and onset latencies of CSD components. Peak amplitudes and latencies at the onset and offset of individual CSD components were determined for each channel. In accordance with Kaur et al. (2004), peaks were defined as the highest amplitude of an individual sink. The problem of accurate onset and offset latency determination has been explained previously (Kaur et al., 2004). We modified their two-point procedure to estimate onset latencies by fitting a line around the point at which each curve surpasses 3 SDs above/below baseline. The intersection of this line with baseline was defined as the onset latency. We compared this procedure with the previously introduced method by Kaur et al. (2004) and found less variance especially at low amplitudes. For control of this automated processing, all plots were additionally inspected by a trained observer and, in a few cases, corrected by hand. All computations were performed with custom-written programs using Matlab. To define the BF, we compared mean peak amplitudes, mean peak latencies, and mean onset latencies of the granular sink and found highly correlated results. The BF was defined as the frequency of the pure tone stimulus in our stimulus set that elicited the maximum peak in the initial granular sink.

Averaged rectified CSD and residue analysis. The averaged rectified CSD (AVREC) (Givre et al., 1994; Schroeder et al., 1998) was calculated by averaging the absolute values of the CSD separately across the $n$ channels for each trial. Subsequently, data were averaged across trials (Eq. 2). Although information about the direction of transmembrane current flow is lost by rectification, the resulting AVREC waveform provides a measure of the temporal pattern of the overall strength of transmembrane current flow (Givre et al., 1994; Schroeder et al., 1998). The relative CSD residues (Harding, 1992) were calculated as the sum of the CSDs over the $n$ channels divided by the sum of the respective absolute values (Eq. 3).

$$
\begin{gathered}
\text { AVREC }=\frac{\sum_{i=1}^{n}\left|\operatorname{CSD}_{i}\right|(t)}{n}, \\
\text { relative residues }=\frac{\sum_{i=1}^{n} \operatorname{CSD}_{i}(t)}{\sum_{i=1}^{n}\left|\operatorname{CSD}_{i}\right|(t)} .
\end{gathered}
$$

For the quantitative analysis of the AVREC and the relative residues, we used single-trial CSD waveforms without spatial filtering. Onset latencies of AVREC and relative residues were defined as the times when the respective waveforms surpassed 3 SDs from baseline. Baseline values were calculated from a $200 \mathrm{~ms}$ time window preceding the stimulus.

Immunohistochemistry and histology. After the acute experiments, animals were killed by intracardial injection of $0.5 \mathrm{ml}$ of T61 (Intervet). Detailed histological analysis of the electrode position relative to the cortical laminae has been performed in five animals. To this end, brains were removed immediately after killing and cryoprotected with $8 \%$ gelatin and conveyed to isopentane cooled to $-50^{\circ} \mathrm{C}$ in a nitrogen bath. Brains were cut on a cryotome into $50-\mu \mathrm{m}$-thick horizontal sections that were collected in phosphate-buffered $0.1 \mathrm{~m}$ saline. Sections were counterstained for cell bodies with cresyl violet staining (Nissl staining). The perpendicular position of the multichannel shaft electrode could be identified by a lack of Nissl substance across cortical layers (supplemental Fig. 2, available at www.jneurosci.org as supplemental material). The Nisslstained sections also allowed relating the laminar depth of each respective multielectrode channel to the corresponding cortical layer in each animal $(n=5)$, despite some variance in thickness of cortical layers across individuals (for details, see supplemental Note 2, available at www.jneurosci. org as supplemental material).

\section{Results}

We have investigated the laminar organization of afferent thalamocortical input and convergent inputs from the intracortical microcircuitry in gerbil primary auditory cortex. Laminar CSD profiles in A1 have been evoked by pure tones using a wide range of frequencies. To dissociate thalamocortical input to primary sensory cortex from horizontal intracortical synaptic interactions, we applied the $\mathrm{GABA}_{\mathrm{A}}$ receptor agonist muscimol alone $(0.2-1 \mu \mathrm{g} / \mu \mathrm{l})$ (Talwar et al., 2001; Kaur et al., 2004) or concomitantly with the $\mathrm{GABA}_{\mathrm{B}}$ antagonist SCH50911 (1:1.5) (Yamauchi et al., 2000) topically onto the cortical surface. We quantified changes of the laminar CSD profile after cortical silencing, which allows insights into the relative contributions, the temporal recruitment, and the laminar organization of afferent thalamocortical input and intracortical input, respectively, to primary auditory cortex (cf. Schroeder et al., 2003; Kaur et al., 2005; Lakatos et al., 2005, 2007; Chen et al., 2007).

\section{Cortical silencing to dissociate thalamocortically and intracortically evoked CSD components}

The pure-tone-evoked spatiotemporal CSD profile in the untreated primary auditory cortex field Al of the gerbil showed a characteristic feedforward pattern, similar to other findings in the visual (Schroeder et al., 1998; Hirsch and Martinez, 2006; Chen et al., 2007) and auditory (Barth and Di, 1990; Steinschneider et al., 1992, 1998; Sakata and Harris, 2009; Szymanski et al., 2009; Atencio and Schreiner, 2010) systems.

The CSD profile after BF stimulation in the untreated auditory cortex (Fig. 1A) indicates feedforward input from afferent thalamocortical projections terminating in granular layers III/IV and also intracortical connections to supragranular and infragranular layers (cf. Schroeder et al., 1998; Chen et al., 2007; Sakata and Harris, 2009; Atencio and Schreiner, 2010). The BF was defined as the tone frequency that evoked the highest peak of the sink in the granular layer. To dissociate the contributions of afferent thalamocortical and intracortical input to this cortical feedforward CSD profile, we used a conventional approach for pharmacological cortical silencing of intracortical connections (Krupa et al., 1999; Yamauchi et al., 2000; Fox et al., 2003; Li and Ebner, 2006; Andolina et al., 2007; Liu et al., 2007; Wu and Yan, 2007) (for further discussion, see below). The rationale of this approach is to use concentrations known to influence exclusively intracortical postsynaptic potentials without limiting axon conductivity (Martin and Ghez, 1999; Edeline et al., 2002; Kaur et al., 2004). Behavioral work has also shown that these concentrations block psychophysical detection of pure tones in rats (Talwar et al., 2001). Experimentally induced hyperpolarization of neurons may abolish their spiking output without affecting the barrages of postsynaptic potentials and therefore neuronal input to the hyperpolarized population (for rationale, see McCormick et al., 2003). Hence, synaptic activities driven by intracortical processes ought to be silenced completely, whereas bottom-up thalamocortical input should not be blocked (Kaur et al., 2004). For a detailed analysis, we have identified distinct components of the spatiotemporal CSD profile (Fig. $1 A$ ). We refer to the initial granular sink as S1, the initial supragranular sink as S2, and infragranular sink as S3. Furthermore, the granular S1 is balanced by a supragranular source So 1 and an infragranular source So3. A granular source occurring at longer latencies and balancing S2 and S3 is called So2. In some examples, we also found an initial short-latency input to layer V, which is referred to as iS1 (for infragranular initial sink). In Figure $1 B--D$, the temporal development of CSD profiles (shown for BF stimulation) reflects the 

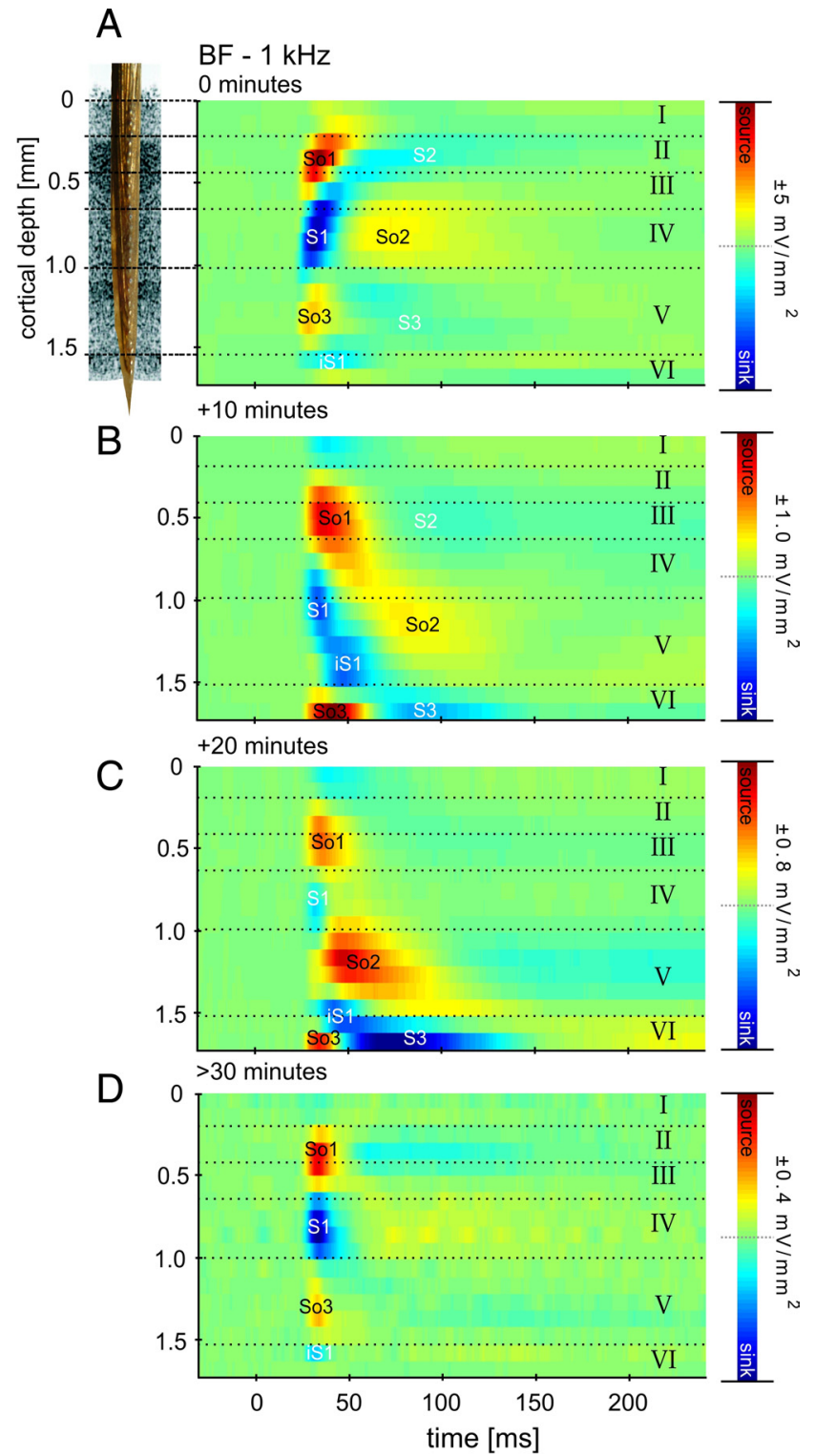

Figure 1. Temporal development of silencing the intracortical contribution of tone-evoked activity in primary auditory cortex. Shown are the CSD profiles across cortical layers (roman numbers) evoked by acoustic stimulation with the BF represented at the measurement site, after different times following topical application of muscimol and SCH50911 to the auditory cortex. Prominent current sources (So1, S02, So3) and current sinks (S1, S2, S3, iS1) are shown in red and blue, respectively. Inset, Custom-made multichannel shaft electrode used for recording. $\boldsymbol{A}$, Canonical laminar feedforward CSD profile in A1 (for details, see Results). $\boldsymbol{B}$, At 10 min after application of the silencing mixture, maximum amplitudes of current sources and sinks were reduced to $20 \%$ of the original values (see color bar scaling), and the spatial pattern is indicative of diffusion of the mixture from the top layers to the infragranular layers. C, At 20 min after application, amplitudes were further reduced. $\boldsymbol{D}$, After $30 \mathrm{~min}$, the tone-evoked CSD pattern has stabilized, and CSD amplitude maxima and minima were $<10 \%$ of the predrug values. Cortical layers were derived from histological analysis of this particular measurement site (supplemental Note 2, animal 1, available at www.jneurosci.org as supplemental material).

drug diffusing through cortex after epidural application. Note the substantial reduction in amplitude (indicated by the scale bar next to each graphic panel) at every time point. After $20 \mathrm{~min}$ following application, the drug had diffused through layer IV, because the initial granular sink S1 was found to be significantly reduced. Consequently, a disinhibition of layer V pyramidal neurons by blocking granular circuits could possibly have caused the strong infragranular sink S3 (S3 is stronger compared with 10
Table 1. Relative ratio (\%) of evoked granular (S1) and extragranular (S2, S3) amplitudes before and after cortical silencing

\begin{tabular}{lcll}
\hline Relative strength of sink S1 to S2/S3 & Untreated & Muscimol & Musicmol + SCH50911 \\
\hline S2/S1 (\%) & & & \\
BF & 33.08 & 6.15 & 9.84 \\
1 octave & 52.17 & 9.25 & 5.81 \\
2 octaves & 109.38 & 2.65 & 1.73 \\
$\quad$ 3 octaves & 151.16 & & \\
S3/S1 (\%) & & & \\
BF & 17.12 & 3.85 & 8.66 \\
1 octave & 20.23 & 6.25 & 2.47 \\
2 octaves & 50.13 & 2.5 & 9.21 \\
\hline
\end{tabular}

Comparative analysis of relative amplitude strength (\%) of the granular sink $\$ 1$ versus extragranular components $\mathrm{S2}$ and $\mathrm{S3}$ (peak amplitudes of $\mathrm{S2}$ and $\mathrm{S3}$, respectively, are divided by peak amplitude of $\mathrm{S1}$ ). In untreated cortex amplitude ratios of $S 1$ compared with $S 2$ and $S 3$ show that relative contributions of extragranular components increase with distance to the BF. After cortical silencing, the relative contributions of the later extragranular components are significantly smaller compared with the robust granular sink. Therefore, the later extragranular sinks and sources after the initial source-sink-source triplet after drug application reflect repolarization currents that are observable because they are not superimposed by other CSD components attributable to active processes in the cortex during later time points after stimulus onset.

min after drug application). S3 was silenced after the drug reached infragranular layers (after $>30 \mathrm{~min}$ ). Note that, $30 \mathrm{~min}$ after application, the drug reached almost all cortical layers, resulting in a concise and robust source-sink-source triplet. This finding shows that we can effectively dissociate tone-evoked thalamocortical and intracortical contributions to the physiological feedforward CSD profile in primary auditory cortex using cortical silencing. We found all later components (S2 and S3) of the cortical response to be evoked through transynaptic intracortical processing (for detailed quantitative analysis, see Table 1) (Kaur et al., 2004). The robust granular sink S1 indicates activation of synapses mainly within layer IV, which is believed to be the major laminar recipient of tonotopically organized afferent input from the ventral part of the medial geniculate body (vMGB) (Budinger et al., 2000a,b; Huang and Winer, 2000; Hackett, 2010). Compared with the activation of the granular layers in untreated A1, the granular sink is spatially restricted mainly to layer IV and to some degree to layer IIIb or layer Va, with no activation of upper layer IIIa or layer II. Hence, activation of upper layer IIIa or layer II could be related to the activation of intracortical recurrent microcircuits (Douglas et al., 1995; Liu et al., 2007). In some cases, we found the infragranular sink iS1 to be present after drug application. This is indicative of a separate thalamocortical projection to layer $\mathrm{V}$ pyramidal neurons, which has been described in different anatomical studies (Budinger et al., 2000a; Huang and Winer, 2000; Linden and Schreiner, 2003; Winer et al., 2005) and a recent physiological study (Szymanski et al., 2009).

\section{Differential contributions of thalamocortical and intracortical inputs in dependence on spectral input}

Using the described cortical silencing method, we aimed at dissociating the contribution of subcortical feedforward input to cortical CSD profiles evoked with different stimulation frequencies from contributions of convergent intracortical input. A qualitative illustration of the acoustically evoked CSD profiles evoked by different frequencies is shown in Figure $2 \mathrm{~A}$. Stimulation with pure tone frequencies in close spectral distance ( $\leq 2$ octaves) to the $\mathrm{BF}$ and at larger spectral distance ( $\geq 3$ octaves) were referred to as nearBF and nonBF stimulation, respectively. We found pure tone frequencies of 1 octave distance to evoke significantly different CSD profiles, indicative of activation of different synaptic populations, in accordance with our previous results on the tono- 


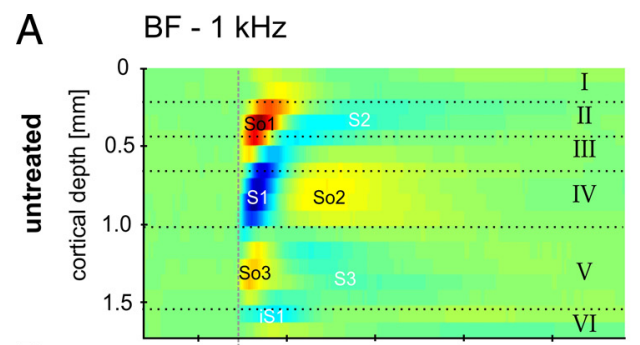

nearBF - $2 \mathrm{kHz}$
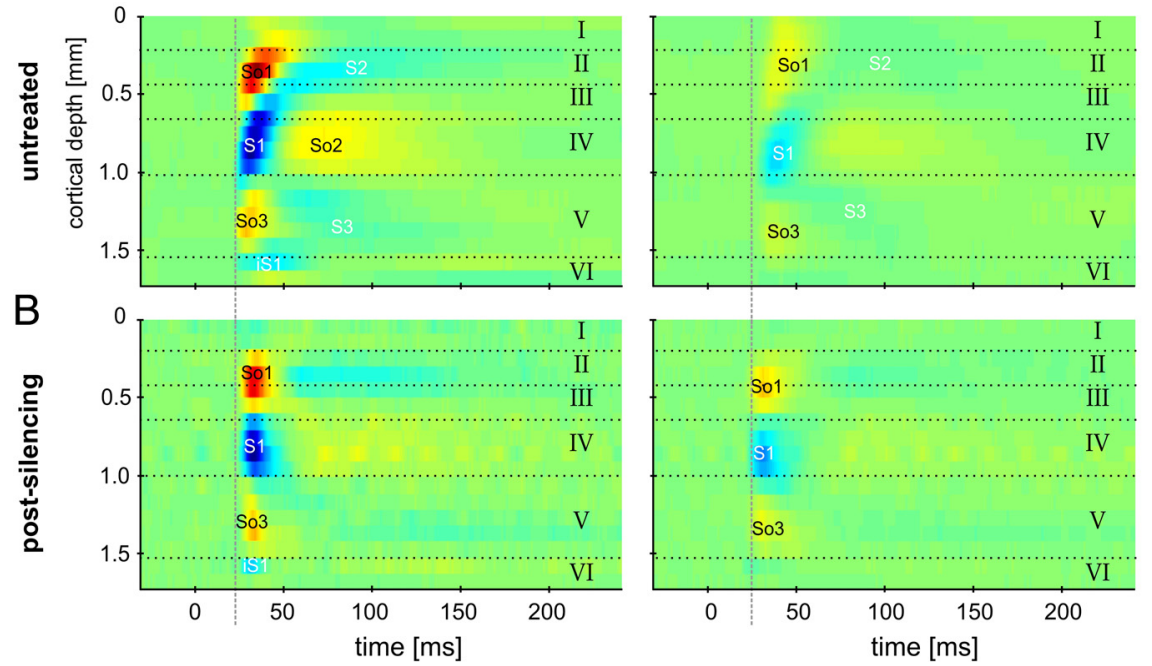

nonBF - $8 \mathrm{kHz}$
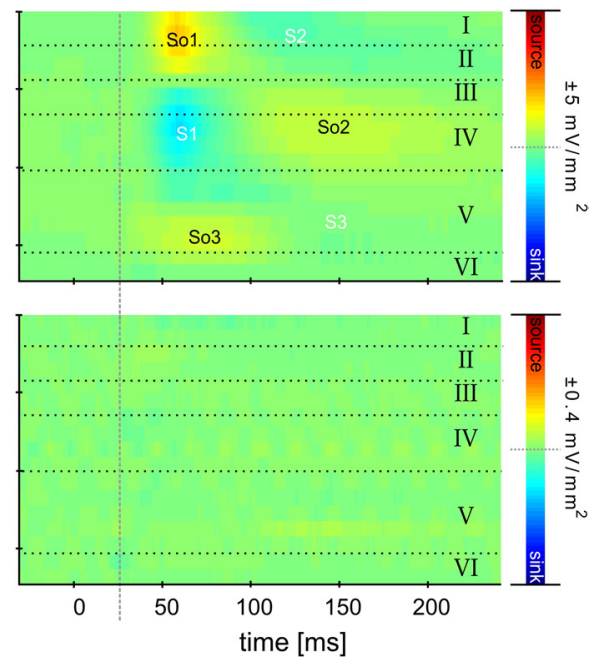

Figure 2. Dissociation of thalamocortical and intracortical contributions of the laminar CSD profile. $A$, Laminar processing in $A 1$ differed with stimulation frequency (BF, nearBF, or nonBF stimulation). $\boldsymbol{B}$, After cortical silencing, remaining sinks were observed exclusively after BF and nearBF stimulation in thalamocortical input layer IV ( $>30$ min) (see Fig. 1). Activations after nonBF stimulation were abolished completely. Note that the onset latency of the nearBF-evoked initial granular sink $\$ 1$ was faster after drug application. In contrast, cortical silencing had no effect on the onset latency of BF-evoked S1 (see dashed line as reference).

topic organization of tone-evoked local field potentials in gerbil primary auditory cortex field A1 (Ohl et al., 2000a,b).

Stimulation with the BF elicited the most profound activation throughout cortical layers (Fig. $2 \mathrm{~A}$ ). Evoked activation of granular and infragranular layers decayed with spectral distance of input from the BF. Spectral tuning width in the sense of significant evoked deflections was found to be $4.8 \pm 1.82$ octaves for infragranular sinks. Granular and supragranular activation showed spectral tuning widths of $6.4 \pm 0.52$ octaves $(\mathrm{S} 1)$ and $6.8 \pm 0.45$ octaves (S2), respectively. In some cases, we even found activation throughout the whole presented frequency range of 8 octaves (up to 4 octaves apart from BF). The granular sink S1 showed fastest onset latency for $\mathrm{BF}$ stimuli and longer latencies for nearBF and nonBF stimulation (Fig. $2 \mathrm{~A}$, dashed lines refer to initial onset latency at BF stimulation). We found the initial response in granular layers regardless of stimulation frequency. Hence, the initial sink in granular layers need not be a unique feature of profiles evoked by afferent thalamocortical input but could also occur in profiles with a considerable amount of initial horizontal intracortical input.

To address this issue, Figure $2 B$ shows the results of CSD profiles evoked with the different stimulation frequencies after cortical silencing. Again, we found all later components (S2 and S3) of the cortical response to be evoked through transynaptic intracortical processing (Table 1). Robust granular sinks after BF and nearBF stimulation were found in layer IV, the major laminar target of afferent input from the vMGB. After cortical silencing, the granular sink showed strongly decreased amplitudes (Fig. 3 ) and was found to have a reduced bandwidth of $3.2 \pm 1.4$ octaves (see Fig. 7) (cf. Kaur et al., 2004). The reduced bandwidth reflects the activation of thalamocortical synapses, which should still be active after cortical deactivation. Therefore, we can conclude that, before cortical silencing, other intracortical synaptic populations in layer IV are recruited in addition to thalamocortical inputs (Krupa et al., 1999; Li and Ebner, 2006; Andolina et al., 2007). In this context, it is worth noting that the lemniscal pathway via vMGB to A1 represents only $\sim 10-20 \%$ of inputs to cortical layer IV (Ahmed et al., 1997; Logothetis, 2008). The ac- tivations evoked by nonBF stimulation were found to be strongly reduced (at least smaller than $0.005 \mathrm{mV} / \mathrm{mm}^{2}$ ). In contrast, electrophysiological monitoring of isolated thalamocortical EPSPs suggested that afferent inputs elicit equally strong amplitudes across stimulation frequencies (Liu et al., 2007). We found activations after $\mathrm{BF}$ and nearBF stimulation to have comparable amplitudes, especially when $\mathrm{GABA}_{\mathrm{B}}$ inhibition was additionally blocked (compare with Fig. $3 A$ ). We therefore conclude that nonBF stimulation frequencies do not activate the local afferent synaptic populations. Notably, we found the onset latency of the nearBF-evoked initial granular sink S1 to be faster after drug application. The same held for the sources Sol and So3. In contrast, cortical silencing did not affect the onset latency of BFevoked S1 (paired $t$ test, $p=0.92$ ) (Fig. 3, dashed line). Thus, cortical silencing had different effects on the responses evoked by $\mathrm{BF}$ and nearBF stimulation. For all robust granular sinks, we observed a temporally very precise and spatially compressed profile as described in Figure 1. In addition, the duration of the granular sink was decreased. Effectively, this means that the latency of monosynaptic thalamocortical input was essentially independent of stimulation frequency (but see Kaur et al., 2004). To quantify the contribution of afferent thalamocortical input to the evoked CSD components of the untreated auditory cortex, we analyzed and compared different sink parameters before and after cortical silencing.

\section{Quantification of CSD components referring to thalamocortical or intracortical inputs}

Before cortical silencing, onset latencies and mean peak amplitudes of the prominent CSD components were analyzed in dependence of spectral input. Sharpest spectral tuning was found for the mean peak amplitude of the granular sink. BF-evoked granular sinks had peak amplitudes with a mean of $-1.11 \pm 0.21$ $\mathrm{mV} / \mathrm{mm}^{2}$. Supragranular sinks had mean amplitudes of $-0.59 \pm$ $0.03 \mathrm{mV} / \mathrm{mm}^{2}$ and infragranular sinks of $-0.20 \pm 0.2 \mathrm{mV} / \mathrm{mm}^{2}$.

Cortical silencing by application of muscimol alone or muscimol plus SCH50911 blocked prominent supragranular and infragranular sinks S2 and S3 (Table 1). For thalamocortical- 


\section{A Peak amplitude tuning}

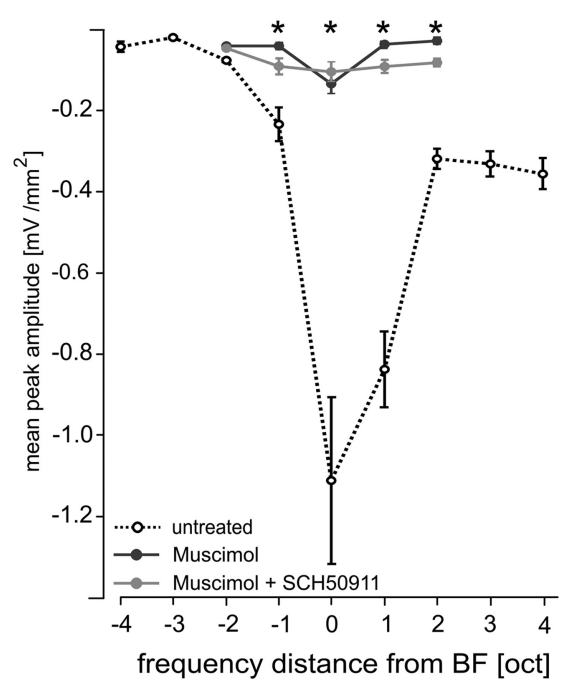

B Onset latency tuning

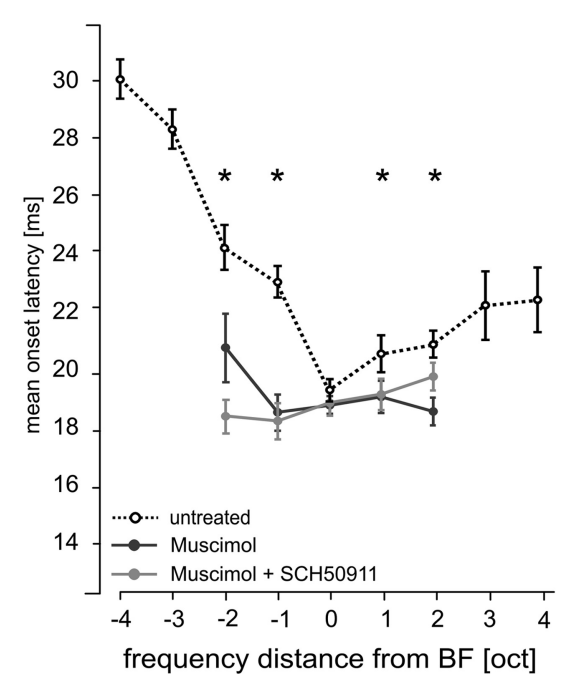

Laminar processing of afferent thalamocortical input and horizontal intracortical inputs

In Figure $4 A$, laminar mean \pm SEM onset latencies are shown for prominent sinks S1-S3 evoked by pure tones of different frequency. The granular sink S1 was faster after BF stimulation than for other stimulation frequencies and showed identical onset latencies from 700 to $1100 \mu \mathrm{m}$ cortical depth. Near/nonBF-evoked S1 in contrast had longer latencies. Furthermore, the onset latency profile showed a faster onset in higher laminae located at layer III and upper layer IV compared with deeper layer IV. In particular, nearBF-evoked S1 showed a spatially broad activation (500-1100 $\mu \mathrm{m})$ but with a temporal delay of activation along the cortical depths. The nonBF-evoked granular sink S1 showed spatial activations from 400 to $900 \mu \mathrm{m}$ cortical depth and a delay of the initial activation in upper layer III of $\sim 2 \mathrm{~ms}$ compared with $\mathrm{BF}$. However, nonBF-evoked S1 did not fully extend into layer $\mathrm{V}$. We conclude that different synaptic subpopulations are responsible for the granular sink S1 evoked by $\mathrm{BF}$, nearBF, and nonBF frequencies. A differential recruitment of intracortical in combination with the $\mathrm{GABA}_{B}$ receptor agonist $\mathrm{SCH} 50911$ to block possible side effects of muscimol on presynaptic processing of thalamocortical input (see Results). $\boldsymbol{A}$, Mean \pm SEM peak amplitudes of the granular sink S1 evoked by pure tones of different spectral distance to the best frequency. Note the significant decreases of amplitude values at the BF and near $B F$ frequencies and the total absence of measurable responses at nonBF frequencies (for quantification of the ratios of decrease, see Table 1) after both silencing methods. $\boldsymbol{B}$, Mean \pm SEM onset latency of granular sink $\mathrm{S} 1$ decreased after drug application selectively for nearBF stimulation but was constant for BF stimulation. In all cases, we did not find significant differences between muscimol $(n=10)$ or muscimol plus SCH50911 ( $n=3)$ induced cortical silencing. ${ }^{*} p<0.001$ indicates significant changes after drug application (paired $t$ test).

Table 2. Relative amplitude decrease of granular sink after both cortical silencing approaches

\begin{tabular}{llll}
\hline & Untreated & Drug condition & \% decrease \\
\hline Muscimol $(0.2-1 \mu \mathrm{g} / \mu \mathrm{l} ; n=10)$ & & & \\
$\quad$ BF & -1.111 & -0.134 & 87.9 \\
$\quad \pm 1$ octave & -0.677 & -0.05 & 92.6 \\
$\quad \pm 2$ octave & -0.233 & -0.041 & 82.5 \\
Muscimol/SCH50911 $(1: 1.5 ; n=3)$ & & & \\
BF & -1.044 & -0.103 & 89.74 \\
\pm 1 octave & -0.796 & -0.107 & 86.51 \\
\pm 2 octave & -0.621 & -0.079 & 87.24 \\
\hline
\end{tabular}

Comparative mean peak amplitudes (millivolts per square millimeters) and relative decrease of the granular sink shown for both pharmacological silencing methods. Note that, although relative decrease is similar in both cases, the amplitudes for nearBF stimulation ( $\pm 1-2$ ocatves) are higher after cortical silencing with muscimol and SCH50911 applied concomitantly.

recipient layer IV, the granular sink was still evoked by pure tone frequencies covering 5 octaves in maximum (BF and nearBF). However, mean peak amplitudes and onset latencies showed prominent changes. For mean peak amplitudes, the amount of decrease was similar for BF and nearBF (Fig. 3A, Table 2). Mean peak amplitude of the BF-evoked granular sink was reduced by $88 \%$ and between 83 and $96 \%$ for nearBF stimulation. However, for mean onset latencies, we found profound differences between BF- and nearBF-evoked S1. Onset latencies after BF stimulation did not show significant changes, whereas nearBF sinks referring to the borders of thalamocortical input decreased in latency (Fig. 3B). The selective drug effect on onset latencies for nearBF stimulation of a cortical site suggests a recruitment of both thalamocortical and intracortical inputs before, and exclusively thalamocortical inputs after, drug application, respectively. connections could in principle be responsible for the described diversity of laminar depths of initial activation. This finding would match anatomical work describing local intracortical connectivity strongly projecting toward upper layers III and II (Ojima et al., 1991; Budinger et al., 2000b; Dantzker and Callaway, 2000; Thomson and Bannister, 2003). In addition, our findings also match previous electrophysiological studies demonstrating different laminar termination depths of horizontal connections as a function of their lateral distance (Luhmann et al., 1991). Layers II/III consequently would provide an important function for intercolumnar information transfer for broad spectral integration. differences between BF and nearBF/nonBF stimulation. It could be inferred how extragranular sinks S2 and S3 originate from different generators of the cortical microcircuitry based on the temporal characteristics of the initial granular sink. After BF stimulation, a consistent progression of activation reaches up to cortical layer I through direct intracolumnar transmission (Bannister, 2005; Sakata and Harris, 2009). For nearBF/nonBF-evoked supragranular sink S2, we found a reversed laminar gradient of delay (i.e., shorter latencies in layer I vs II), potentially reflecting different laminar inputs for nearBF/nonBF stimulation compared with $\mathrm{BF}$ stimulation. One interpretation could be that additional input from intercolumnar horizontal projections terminates in supragranular layers. This input would emanate from cortical sites for which the presented frequency is the BF and hence would be independent from intracolumnar activations after BF stimulation. This is in line with the observation that mean peak amplitudes of S2 are less strongly modulated by stimulation frequency than S1 (mean peak amplitudes of S2 evoked with stimulation: $\mathrm{BF}, 0.37 \pm 0.05 \mathrm{mV} / \mathrm{mm}^{2}$; nearBF, $0.31 \pm 0.1 \mathrm{mV} / \mathrm{mm}^{2}$; and nonBF, $0.28 \pm 0.02 \mathrm{mV} / \mathrm{mm}^{2}$ ). Activations of infragranular lay-
Subsequent extragranular activations also showed profound 
ers V and VI ( sink S3) were found exclusively as a subsequent effect of extension of $\mathrm{S} 1$ into upper layer $\mathrm{V}$, which occurred after stimulation with $\mathrm{BF}$ or nearBF frequencies.

CSD profiles were previously discussed predominantly with respect to EPSPs (Mitzdorf, 1986), although inhibitory synapses could in principle add a direct synaptic contribution to extracellular sources. Nevertheless, CSD analysis yields at least an indirect measure of inhibitory influences in that CSD profiles originate from the interaction of excitation and inhibition. The interaction of sinks and sources obey the law of superposition. Changes in stimulus parameters (such as stimulation frequencies) could cause a differential recruitment of relative contributions of specific synaptic populations, leading to equivalent shifts of the related sink-source components. In this respect, it has been demonstrated in gerbil auditory cortex that horizontal connections terminate on both local inhibitory interneurons and excitatory pyramidal neurons (Kurt et al., 2008; Moeller et al., 2010), which could lead to sideband inhibition or facilitation, respectively. Hence, the different sink-source relations revealed with $\mathrm{BF}$ or nonBF frequency stimulation (compare with Fig. $4 A$ ) in this study could be attributable to different types of interaction between excitation and inhibition.

Cortical silencing blocked longerlatency extragranular sinks (supplemental Figs. S2, S3, available at www.jneurosci. org as supplemental material) completely, which indicates that these layers receive mainly intracortical projections (Table 1). Cortical silencing of the intracortical microcircuitry significantly affected the amplitudes and latencies of S1 (compare with Fig. 3). This finding is attributable to the fact that hyperpolarization of the neuronal populations in the granular layers prevents afferent inputs from efficiently recruiting the recurrent intracortical microcircuitry (Liu et al., 2007). Monosynaptic afferent input causes a local net hyperpolarization of the extracellular field near the thalamocortical recipient population, resulting in a local sink (McCormick et al., 2003). As stated above, the granular layers are the most likely target region of convergent thalamocortical (5-20\%) and intracortical (80-95\%) projections. Nevertheless, it is still not clear whether the intracortical synapses are related to local intracolumnar recurrent microcircuits or to lateral connections between cortical columns, as has been proposed based on anatomical data (Ojima et al., 1991, 1992; Budinger et al., 2000b). To disentangle the putative contributions of different intracortical synaptic populations to granular activations, the drug-induced changes of the $\mathrm{S} 1$ were analyzed. Figure $4 B$ shows the drug-induced changes of

\section{Laminar mean onset latencies}
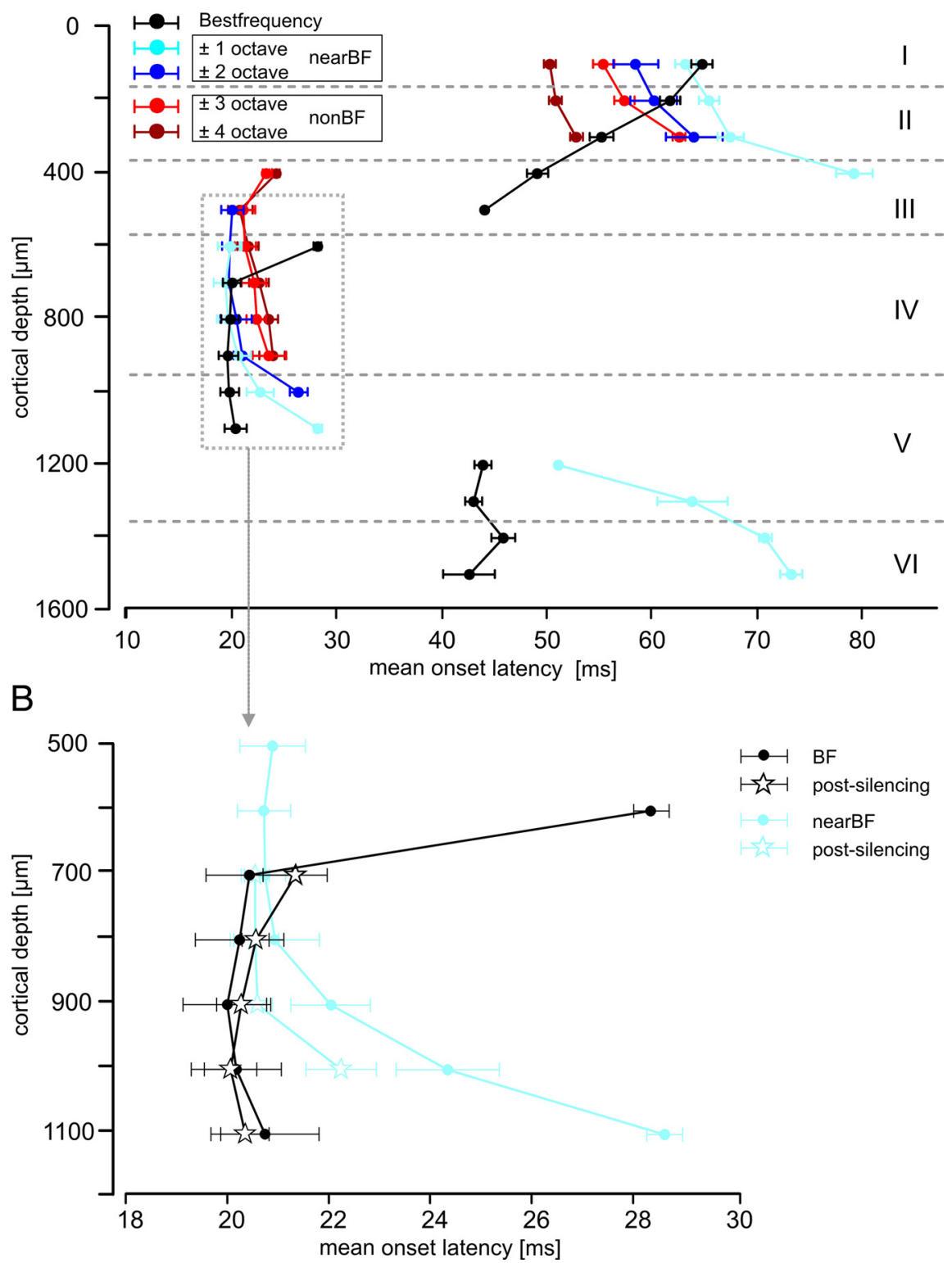

Figure 4. Laminar processing of convergent afferent thalamocortical input and intracortical input. $\boldsymbol{A}$, Mean \pm SEM onset latencies are shown for the three main sinks ( $1,400-1100 \mu \mathrm{m} ; \mathrm{S2}, 0-500 \mu \mathrm{m} ; \mathrm{S3}, 1200-1600 \mu \mathrm{m})$ evoked with BF, nearBF, or nonBF stimulation. $\boldsymbol{B}$, After cortical silencing, the granular sink $\mathbf{S 1}$ was still measurable. Shown are the mean \pm SEM onset lencies for this sink, evoked by BF and nearBF frequencies (pooled results from $\pm 1-2$ octaves distance from BF) before and afte cortical silencing. Note that cortical silencing affected the spatial and temporal structure of the granular sink selectively after stimulation with nearBF stimuli. Cortical layers indicated here were derived from histological analysis of five different measurement sites and represent the mean relations (supplemental Note 2, available at www.jneurosci.org as supplemental material).

the S1 laminar mean onset latency in greater detail. Onset latencies after BF stimulation are plotted in black and after nearBF stimulation (pooled $\pm 1-2$ octaves from $\mathrm{BF}$ ) in light blue. Activations by nonBF stimulation are not depicted in Figure $4 B$, because they were completely blocked after cortical silencing. After BF stimulation, S1 did not show a significantly altered onset latency (paired $t$ test, $p=0.92$ ) but was spatially restricted to cortical depths of $700-1100 \mu \mathrm{m}$. This could result from blocked local recurrent microcircuits between layers IV and III after pharmacological silencing (Liu et al., 2007). After nearBF stimulation, cortical silencing shifted the laminar depth in which initial activation occurred from upper layer IV and layer IIIb (450-700 
$\mu \mathrm{m})$ to deeper layer IV and layer Va $(700-1000 \mu \mathrm{m})$. The shortlatency initial current sink in upper layers IV and IIIb was completely blocked by silencing indicating its origin from intracortical input. Deeper layer IV and layer Va $(700-1000 \mu \mathrm{m})$ showed faster (1-2 ms) latencies after silencing. Hence, cortical silencing caused the different laminar profiles of activation by BF and nearBF stimuli to become more similar to each other.

Our data indicate that initial activation of BF-evoked S1 was mainly based on afferent thalamocortical input because neither its onset latency nor its laminar profile was affected by cortical silencing. In contrast, nonBF-evoked S1 might be attributed to excitatory horizontal synaptic connections because of its complete abolishment by cortical silencing. For intermediate spectral distances to the $\mathrm{BF}$, we found that nearBF-evoked granular responses result from a convergence of both thalamocortical and intracortical inputs in cortical depths of 700-900 $\mu \mathrm{m}$. The faster (1-2 ms) latencies of responses in layers IV and Va (700-1000 $\mu \mathrm{m})$ after cortical silencing indicate that neuronal responses to nearBF stimuli result from a spatially highly organized and temporally very precise recruitment of both convergent thalamocortical and intracortical inputs.

Hence, a number of different input systems apparently contribute to spectral integration in primary auditory cortex (supplemental Fig. 3, available at www.jneurosci.org as supplemental material): (1) afferent thalamocortical input to granular layers (Budinger et al., 2000a; Huang and Winer, 2000; Hackett, 2010), (2) intracolumnar excitation through local intracortical recurrent microcircuits (Liu et al., 2007), and (3) convergent local horizontal intracortical connections between neighboring columns (Ojima et al., 1991, 1992; Budinger et al., 2000b). (4) Additionally, long-range horizontal connections potentially contribute to the edges of the subthreshold receptive field via excitatory connections (Kadia and Wang, 2003; Kurt et al., 2008; Moeller et al., 2010). Although existing data already indicate a division of labor between thalamocortical and intracortical input systems for processing $\mathrm{BF}$ and nonBF stimuli, respectively, it is not fully understood how spectral integration is achieved in the nearBF region in which thalamocortical and intracortical input systems have their greatest overlap. Initial nearBF-evoked responses thus appear to result from a precise, spatiotemporally organized recruitment of both afferent thalamocortical and intercolumnar local horizontal inputs. To critically test this hypothesis, we used a newly developed analysis of laminar CSD profiles aiming to determine whether the initial input is achieved via thalamocortical input or horizontal intracortical input to a cortical site.

\section{Temporal precision of afferent and horizontal convergent input}

Additional insight into the functional convergence of thalamocortical and horizontal intracortical contributions was obtained from the analysis of the AVREC, as a measure of the temporal current flow of overall evoked activity (Givre et al., 1994; Schroeder et al., 1998) and of the relative residues of the CSD (Harding 1992) (for definition, see Materials and Methods). This analysis is based on the following rationale. It was indicated that the activation of presynaptic terminals with its accompanying massive calcium influx contributes significantly to the LFP (Tenke et al., 1993; Stoelzel et al., 2008). Therefore, the primarily orthogonal orientations of thalamocortical and intracortical input systems can be expected to contribute differently to the relative residues of the CSD as measured with a linear electrode array oriented perpendicular to the cortical surface (Fig. 5A). Specifically, it can be hypothesized that activation of the horizontal intracortical input contributes more to the relative residues than activation of the thalamocortical input (see schematic traces in Fig. $5 A$ ). This can be expected because, for lateral connections, current sources and sinks that cancel each other out are more likely to be distributed beyond the cylinder surrounding the electrode array in which extracellular currents would most contribute to the measured local field potential (see schematic depiction of current source and sink distributions in Fig. $5 A$ ). Following this argument, it is reasonable to conclude that the activation of intracolumnar recurrent microcircuits would not contribute to the relative residual of the CSD for two reasons. First, these microcircuits are spatially restricted within a cortical column. Second, they are based on pyramidal neurons (Douglas et al., 1995) whose activation leads to prominent sink-source dipoles and should therefore be detectable with CSD analysis (for additional rationale, see supplemental Note 1, available at www.jneurosci.org as supplemental material). To test this hypothesis and evaluate its usefulness to dissociate thalamocortical, as well as intracolumnar activation specifically from the contributions of horizontal inputs to the evoked activity, we analyzed the temporal relationship of the tone-evoked AVREC waveform and the relative residues of the CSD before and after cortical silencing for BF, nearBF, and nonBF stimuli. Figure 5, $B$ and $C$, shows a representative example. Before cortical silencing (Fig. 5B), we found markedly different latency profiles of AVREC and relative residues dependent on stimulus condition. For BF stimulation $(0.5$ and $1 \mathrm{kHz}$, producing maximum AVREC amplitude), AVREC onset latency was shortest and increased for nearBF and nonBF stimulation (red dots indicate the points in time when the signal crossed the $3 \mathrm{SD}$ level of the prestimulus amplitudes). In contrast, relative residues occurred with shorter onset latencies for nearBF stimulation and nonBF stimulation (see 0.25 or $2-16 \mathrm{kHz}$ ). Above we showed evidence that activation after BF stimulation is a good model for initial thalamocortical input, whereas nonBF stimulation occurred exclusively through horizontal intracortical pathways, producing the situation diagrammed in Figure $5 A$ at the response onset. In other words, AVREC leads the relative residues in time for BF stimulation, whereas AVREC and the relative residues show equal onset latencies for nearBF/nonBF stimulation. After cortical silencing (Fig. 5C), AVREC was greatly reduced, but significant amplitudes were found for a narrow band of stimulation frequencies in the BF region (for quantification, see Fig. 7). In agreement with the hypothesis, relative residues were completely abolished by silencing of intracortical inputs.

Figure 6 shows the quantitative analysis, based on the entire dataset, for the temporal relationship of AVREC and relative residues, allowing inferences regarding all synaptic inputs or only synaptic inputs from intracortical sources, respectively. Mean \pm SEM onset latencies of AVREC and relative residues were found to be faster $(7.75 \pm 0.55 \mathrm{~ms})$ after BF stimulation (paired $t$ test, $p=0.0006$ ) but were not significantly different after nearBF $(p=0.38)$ or nonBF $(p=0.58)$ stimulation (Fig. $6 A)$. This is true for a short time window after onset of evoked activity in which local processes, parallel to the recording axis (thalamocortical input), lead horizontal processes exclusively after BF stimulation. AVREC latency was significantly faster after BF stimulation compared with nearBF stimulation ( $p=$ $0.001)$ and nonBF stimulation $(p=0.0007)$. Interestingly, relative residues were significantly faster after nearBF stimulation compared with BF stimulation $(p=0.024)$ but not after 
A

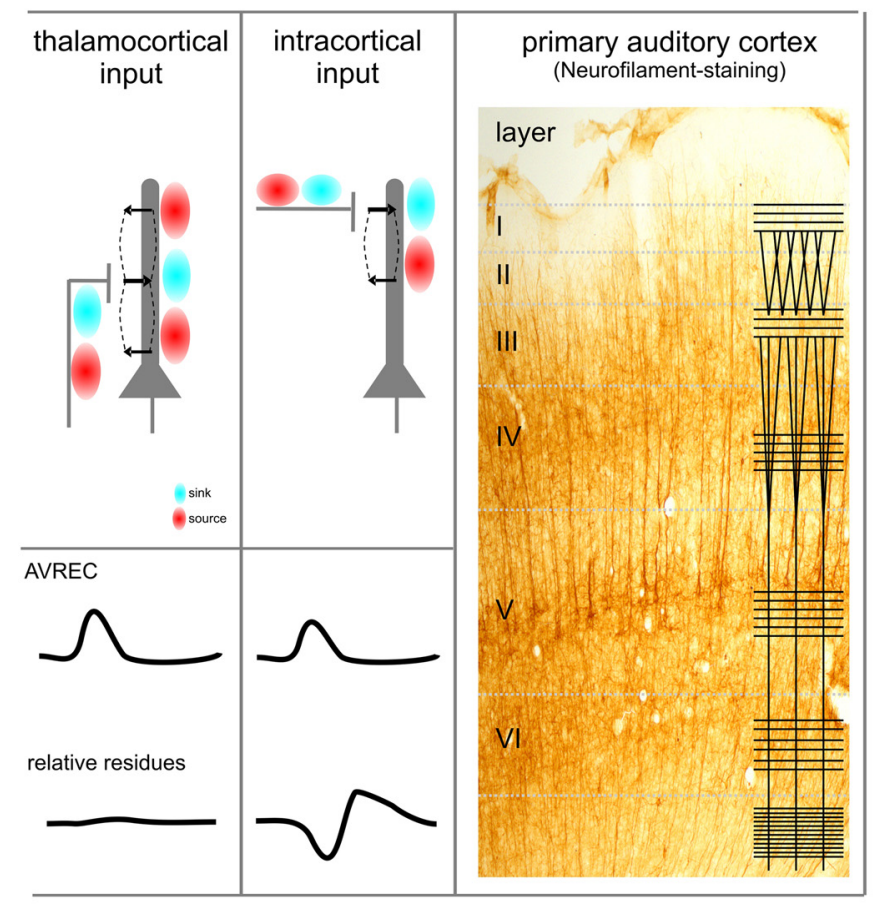

B

untreated

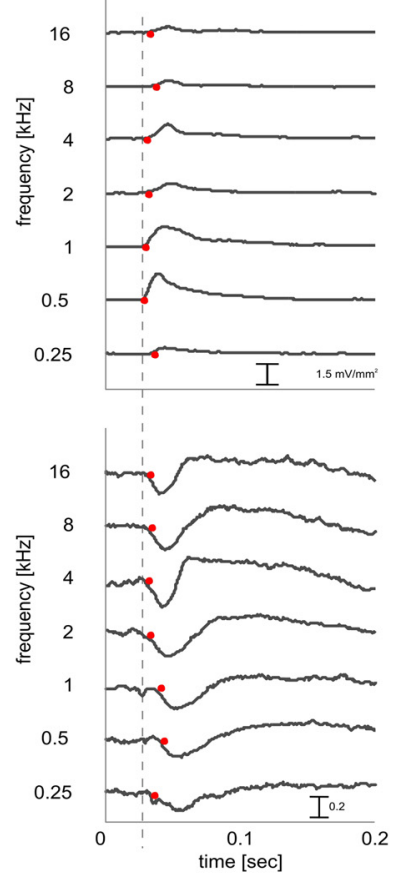

C post-silencing $>60 \mathrm{~min}$

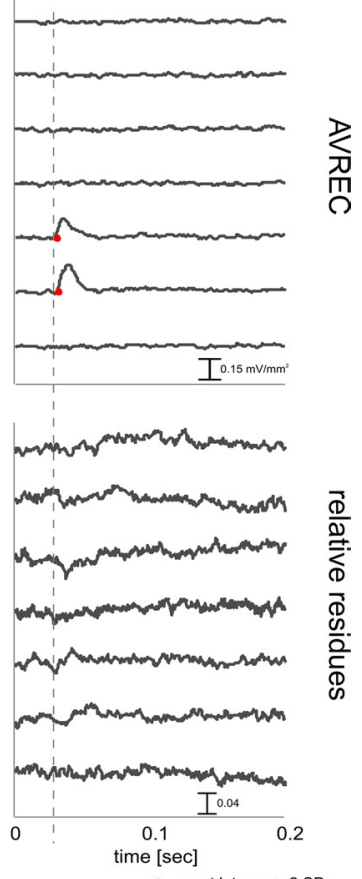

Figure 5. Using the residual CSD for dissociating thalamocortical and intracortical contributions to the laminar activation profile. $A$, Left, The AVREC waveform reflects the temporal characteristics of the overall current flow at a given measurement site (Givre et al., 1994; Schroeder et al., 1998). Relative residues of the CSD reflect the amount of unbalanced sinks and sources (Harding, 1992). The mutually essentially orthogonal orientations of thalamocortical and intracortical input systems can be expected to contribute differently to the relative residues of the CSD measured with a linear electrode array oriented perpendicular to the cortical surface (see traces). Right, Visualization of the orthogonal thalamocortical and intracortical fiber orientation using SMI32 neurofilament staining of gerbil primary auditory cortex (courtesy of Dr. Eike Budinger, Leibniz Institute for Neurobiology, Magdeburg, Germany) reflecting the canonical pattern of fiber orientations in sensory neocortex (overlaid line schematic following Creutzfeldt, 1983). For additional explanation, see Results. $\boldsymbol{B}$, AVREC (top) and relative residues of the CSD (bottom) after stimulation with pure tones before drug application. Stimulation with frequencies around the BF of the site (0.5-1.0 kHz) evoked maximal AVREC amplitudes and minimal AVREC latencies (red dots indicate amplitude values $>3$ SD of the baseline variation). Note that, for these frequencies, the latencies of the AVREC are shorter than the latencies of relative residues, indicative of the dominant thalamocortical drive (dashed line). No such latency differences were found for stimulation frequencies more distant from the BF, indicative of substantial intracortical contributions. C, After pharmacological silencing of all intracortical (namely, horizontal) contributions, tone-evoked relative CSD residues were totally abolished. AVREC amplitudes were measurable after stimulation with frequencies around the BF; AVREC onset latency was not affected.

nonBF stimulation $(p=0.173)$. This result indicates that horizontal inputs are differentially recruited by nearBF/nonBF stimulation compared with BF stimulation and, furthermore, that horizontal inputs contribute to the initial activation of a cortical column when stimulation frequency is not the BF. The hypothesis that relative residuals reflect intracortical inputs exclusively and that the AVREC provides a reliable parameter for estimating the absolute temporal current flow (Givre et al., 1994; Schroeder et al., 1998) can be tested with the cortical silencing approach. Figure $6 B$ shows the mean \pm SEM onset latency of AVREC after cortical silencing (>60 min after drug application). We found BF-evoked AVREC onset latencies not to be affected ( $p=0.71$ ). After cortical silencing, AVREC onset latencies after nearBF stimulation did not show a significant difference compared with BF stimulation ( $p=0.43$ ). Nevertheless, nonBF stimulation did not evoke significant AVREC values, and significant relative residues could not be observed in any case.

Eventually, we showed that potentially anatomically overlapping afferent thalamocortical and intracortical pathways are recruited in a way such that both input systems interact and shape the initial response in primary auditory cortex in dependence on the stimulation frequency. Specifically, horizontal inputs terminating in the (upper) granular layers (compare with Fig. 4) interact directly with afferent inputs when the stimulation frequency is not the BF. In the latter case, afferent input activates a cortical site without convergent horizontal interactions.

\section{Long-range horizontal projections expand the available spectral information of a cortical site}

To what extent intracortical pathways contribute to the information represented at a cortical site about the stimulus spectral energy remains a matter of debate (Matsubara and Phillips, 1988; L. M. Miller et al., 2001b; Kaur et al., 2004; Liu et al., 2007). Therefore, we analyzed the averaged \pm SEM response bandwidth of cortical responses in $\mathrm{A} 1$ (stimulation $20 \mathrm{~dB}$ above threshold) for different parameters derived from our dataset. We found the initial granular sink S1, the AVREC waveform, and relative residues showed similar averaged response bandwidth before cortical silencing (Fig. 7). Response bandwidths of the untreated state $(n=13)$ were compared with response bandwidths after cortical silencing with muscimol $(n=10)$ or muscimol plus SCH50911 $(n=3)$. In both cases, cortical silencing resulted in a significantly decreased bandwidth of spectral tuning of the granular sink S1, as well as the AVREC. Relative residues were blocked completely after silencing. We point out that the additional blockade of $\mathrm{GABA}_{\mathrm{B}}$ inhibition by SCH50911 did not appear to differently affect the still active afferent pathways to sensory cortex (see below). This is in line with previous studies reporting that horizontal intracortical contributions to primary auditory cortical 
A

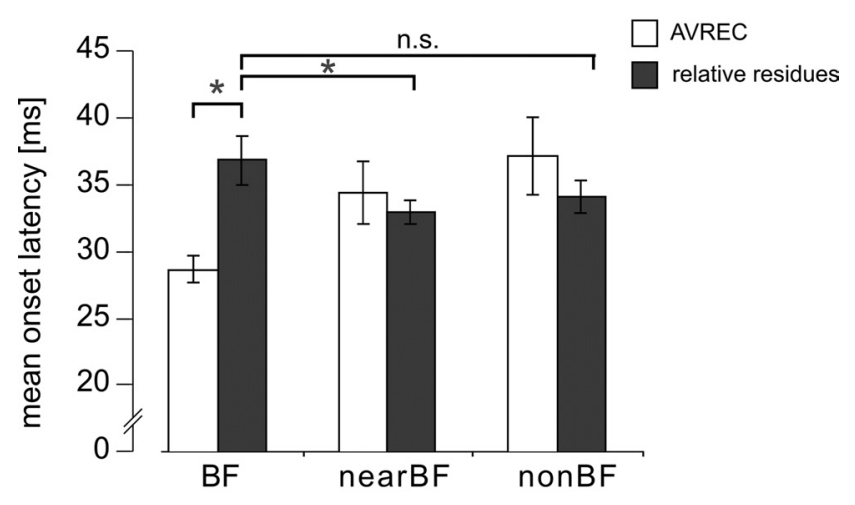

B

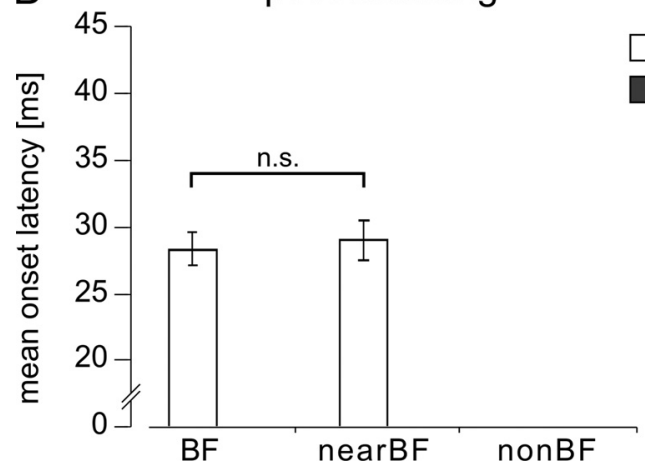

Figure 6. Comparison of mean \pm SEM onset latencies of AVREC and relative residues. $A$, Mean \pm SEM onset latencies of AVREC were significantly shorter for BF stimulation (paired $t$ test, $p=0.0006$ ), but AVREC and relative residues showed no significant differences for near $B F / n o n B F$ stimulation. Mean $\pm \mathrm{SEM}$ onset latencies of relative residues showed significant differences for $B F$ versus nearBF stimulation ( $p=0.023$ ) but were not significantly different for BF versus nonBF stimulation ( $p=0.173$ ). $B$, Cortical silencing blocked all tone-evoked relative residues. Significant AVREC values vanished also for nonBF stimulation. AVREC mean onset latencies for BF and nearBF stimulation showed no significant difference $(p=0.43)\left({ }^{*} p<\right.$ 0.05 , paired $t$ test). n.s., Not significant.

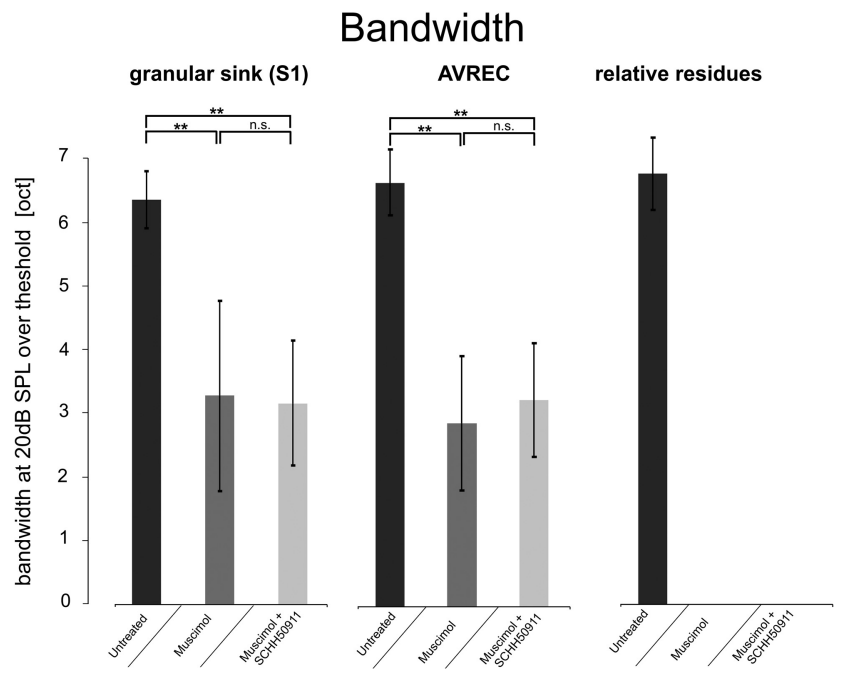

Figure 7. Averaged \pm SEM response bandwidths of the granular sink S1 amplitude, the AVREC, and the relative CSD residues, after stimulation with pure tones $(+20 \mathrm{~dB}$ with regard to threshold at BF) before and after cortical silencing. Values from the untreated cases ( $n=13)$ were compared with cases applying muscimol $(n=10)$ and muscimol plus SCH50911 ( $n=3)$. Cortical silencing resulted in a decreased response bandwidth of the granular sink $\mathrm{S1}$, as well as the AVREC. Relative residues were blocked completely after silencing. ${ }^{* *} p<0.0001$ indicates significant changes after drug application (paired $t$ test). n.s., Not significant. responses lead to a broader response bandwidth compared with thalamocortical inputs. Thus, the available spectral information of a cortical site is expanded through (possibly) subthreshold input through long-range intracortical pathways (cf. Kadia and Wang, 2003; Kaur et al., 2004; Metherate et al., 2005; Moeller et al., 2010).

\section{Adequacy of different pharmacological cortical silencing techniques}

In this study, we used pharmacological cortical silencing by surface application of the $\mathrm{GABA}_{\mathrm{A}}$ agonist muscimol $(4 \mathrm{~mm})$ via craniotomy $\left(\sim 3 \times 4 \mathrm{~mm}=12 \mathrm{~mm}^{2}\right)$ with or without applying a $\mathrm{GABA}_{\mathrm{B}}$ antagonist (SCH50911, $6 \mathrm{~mm}$ ) with previously suggested relative concentrations of both drugs (cf. Yamauchi et al., 2000). Drugs diffused across all cortical layers $(\sim 1.5 \mathrm{~mm})$, and, hence, the affected tissue volume was at least $18 \mathrm{~mm}^{3}$. We did not observe significant differences of response suppression of the laminar CSD profiles using different concentrations or drug mixtures (Fig. 3) and found no significant difference of spectral response bandwidth with or without blocking $\mathrm{GABA}_{\mathrm{B}}$ receptors (Fig. 7). Thus, we conclude that $\mathrm{GABA}_{\mathrm{B}}$-mediated inhibition does not modulate tuning bandwidth in our experiments. A major difference between both silencing approaches was the time course of response suppression. Silencing of intracortical contributions to evoked responses with muscimol alone lasted longer than $10 \mathrm{~h}$ $(n=5)$ but recovered on a much faster timescale when $\mathrm{GABA}_{\mathrm{B}}{ }^{-}$ mediated inhibition was blocked; here a full recovery of the CSD profiles was found after $2-4 \mathrm{~h}(n=3)$. Because the local tissue concentration of any pharmacological agent depends on diffusion speed, we contrasted our baseline data with data obtained 30 min after cortical silencing (Fig. 1). Recently, Liu et al. (2007) used intracortical microinjection of both drugs and observed a spherical diffusion radius of the drugs of at most $600 \mu \mathrm{m}$, resulting in an affected tissue volume of $\sim 1.0 \mathrm{~mm}^{3}$. Furthermore, the experiments were conducted within $30 \mathrm{~min}$ from drug injection, which could lead to significantly different drug concentrations in situ. This might explain the finding of differences between both silencing approaches and the longer recovery period of up to $7 \mathrm{~h}$ instead of $2-4 \mathrm{~h}$. Future studies therefore might choose to use the combination of both drugs to conduct a temporally restricted, and reversible, deactivation of intracortical spiking. Nonetheless, our results show that conclusions derived by past studies using muscimol alone to separate afferent inputs from the contribution of the intracortical microcircuitry can still be considered appropriate.

\section{Discussion}

Neuronal input to auditory cortical neurons can have a thalamic origin or stem from other cortical neurons, and both input systems have been demonstrated to contribute information about the spectral structure of a stimulus to a given cortical site (Schreiner et al., 2000; Edeline, 2003; Metherate et al., 2005). We combined laminar CSD analysis with pharmacological silencing of cortical activity and analysis of the residual CSD to dissociate the feedforward thalamocortical contribution and the intracortical contribution to spectral integration in primary auditory cortex A1. This approach uncovered a mechanism of temporally highly precise integration of both input systems in the nearBF region, in which thalamocortical and intracortical connections have their greatest overlap. 


\section{Thalamocortical and intracortical input systems in primary auditory cortex}

A1 receives a grossly topographically organized afferent input from thalamic nuclei (Merzenich et al., 1975; Velenovsky et al., 2003; Lee and Winer, 2005; Bandyopadhyay et al., 2010; Rothschild et al., 2010). These anatomical connections are much broader, as the fine suprathreshold spectral tuning of a cortical site in A1 would suggest (Wehr and Zador, 2003; Bitterman et al., 2008). Notwithstanding thalamocortical recipient neurons receive their strongest afferent input from vMGB neurons with matched frequency tuning (supplemental Fig. 3, blue arrows, available at www.jneurosci.org as supplemental material). Therefore, cortical tuning is not exclusively determined by afferent feedforward projections (L. M. Miller et al., 2001a; Read et al., 2001; Winer et al., 2005), but intracortical connections endow additional spectral input to a cortical site (Wallace et al., 1991; Schreiner et al., 2000; Kadia and Wang, 2003; Wu et al., 2008). Anatomical frameworks of spectral integration accounting for long-range excitatory horizontal pathways (Kaur et al., 2004) and functionally long-range inhibitory interactions (Tomioka et al., 2005; Kurt et al., 2008; Moeller et al., 2010) have been proposed for the auditory cortex that might allow spectral integration at one cortical site over a large part of the audible spectrum (Metherate et al., 2005) (supplemental Fig. 3, long orange arrows, available at www.jneurosci.org as supplemental material). Recently, intracolumnar recurrent excitation was proposed to selectively amplify suprathreshold activation of thalamocorticalrecipient neurons, thereby sharpening cortical spectral tuning (Liu et al., 2007). These microcircuits operate through cortical neurons in layer II/III, which might not receive any afferent input (supplemental Fig. 3, green circles, available at www.jneurosci. org as supplemental material). Consequently, cortical frequency tuning arises from the interaction and convergence of different subsets of concurring afferent, local, and long-range intracortical inputs. The spatial and temporal interaction of these different pathways during sound processing is still a matter of debate (L. M. Miller et al., 2001b; Imaizumi and Schreiner, 2007).

\section{Temporal precision of horizontal and afferent inputs in thalamocortical-recipient layers}

Here, we have shown that, in addition to these long-range pathways, local horizontal inputs are recruited to complement the input systems described above. The analysis of spatiotemporal CSD profiles and the relative residuals of the CSD in pharmacologically silenced cortex revealed a temporally precise interaction between afferent and horizontal synaptic populations. During BF stimulation, thalamocortical synaptic populations are the first to be activated (compare with Fig. 6A). However, when a cortical site is stimulated with a frequency that is close to the $\mathrm{BF}$ (nearBF), horizontal connections targeting the granular layers arrive from the cortical site for which the stimulation frequency is the BF. We found that these local horizontal inputs have a different laminar organization compared with the thalamocortical projection and contribute to the initial activation of a nearBF-evoked response (Fig. $4 B$ ). Because both synaptic populations terminate in granular layers (cf. Ojima et al., 1991; Budinger et al., 2000a), they still might target the same neurons or at least strategically advantageous positions of the same cortical microcircuits. NearBF stimulation led to a different laminar origin of granular activation (layer IIIa) as thalamocortical input (mainly layer IV, in part layer IIIb or Va) (Fig. 4B). This clearly indicates that excitatory horizontal inputs between neighboring cortical columns traversing through layer II/III (Dantzker and Callaway, 2000; Thomson and
Table 3. Difference of peak amplitudes of prominent sources So 1 and $\mathrm{S}_{3} 3$

\begin{tabular}{lllr}
\hline Amplitude difference (S03 - So1) & BF & 土1 octave & 土2 octaves \\
\hline Untreated & 0.7399 & 0.4845 & 0.2797 \\
After silencing & 0.0221 & 0.0013 & -0.0055 \\
\hline
\end{tabular}

Stronger peak amplitude of the supragranular source S01 compared with the infragranular source So3 for BF and nearBF stimulation is strongly reduced after drug application. Values are shown in millivolts per square millimeters (So3 is subtracted from So1).

Bannister, 2003) contribute to the spectral information available at a cortical site (supplemental Fig. 3, short orange arrows, available at www.jneurosci.org as supplemental material). The decrease in onset latency after cortical silencing during nearBF stimulation indicates that local horizontal inputs have modulatory influences on adjacent cortical sites as well (supplemental Fig. 3, purple arrows, available at www.jneurosci.org as supplemental material). The local horizontal inputs described in this scenario precisely orchestrate the time point of the feedforward laminar activation cascade (Schroeder et al., 1998; Sakata and Harris, 2009) and determine the response latency in relation to the input frequency (when not the BF). Horizontal inputs, therefore, aid to code spectral information via a temporally precise modulation of the sensitivity of adjacent cortical sites to thalamocortical input.

Several mechanisms could contribute to the finding that local horizontal inputs modulate the responses to afferent inputs between cortical columns in a temporally precise manner. What remains open is whether the modulating inputs are based on lateral cortical inhibitory circuitry. One possible mechanism controlling sensory integration is thalamocortical feedforward inhibition (Swadlow, 2002; Swadlow et al., 2002; Gabernet et al., 2005; Cruikshank et al., 2007). Thalamocortical afferents, terminating on both excitatory pyramidal neurons and inhibitory fastspiking interneurons (K. D. Miller et al., 2001), could have invoked intracortical feedforward inhibitory circuits after nearBF stimulation. This was recently suggested to be responsible for lateral sharpening of spectral tuning (Wu et al., 2008). Thalamocortical EPSPs of adjacent cortical sites could therefore be modulated by horizontal inputs mediating a precisely timed and balanced inhibition (Wehr and Zador, 2003; Tan et al., 2004; Razak and Fuzessery, 2006; Wu et al., 2008) that rapidly suppresses excitatory afferent input to non-optimal stimulation. These horizontal inputs can be either directly inhibitory or modulate the cortical responsiveness of adjacent cortical sites through orchestration of local inhibitory circuits (Kurt et al., 2008; Wu et al., 2008; Moeller et al., 2010). Alternatively, excitatory horizontal input can modulate the responsiveness of adjacent cortical sites exclusively through facilitative combinations (Razak and Fuzessery, 2008). Even intrinsic, nonlinear neuronal properties (spike threshold, saturation, etc.) might be sufficient to create such selectivity (Tan et al., 2004; Priebe and Ferster, 2008).

Whether additional inhibitory synaptic inputs play a role for the described intercolumnar interactions of convergent afferent and horizontal projections might not be addressable with classical CSD methods. Nevertheless, we found the lateral microcircuitry of primary auditory cortical thalamocortical-recipient layers to possess an important feature for precise spatiotemporal integration of sensory information across different cortical sites based on interaction of driving and suppressing inputs (cf. Sutter et al., 1999).

\section{Local intracolumnar processing amplifies sensory input} Current sink activation after cortical silencing was mainly reduced to layer IV (S1) or layer V (iS1) and decreased in amplitude 
and duration. Hence, intracortical microcircuits provide a relay of incoming afferent input to be amplified and subsequently fully extend through cortical layers. Cortical amplification of sensory input attributable to recurrent excitatory microcircuitry was mainly described in visual and somatosensory cortex (Douglas and Martin, 2007). The drug-induced massive reduction of S1 mean peak amplitudes (Fig. 4A, Table 2) supports the idea that sensory input is amplified in A1 by means of intracortical recurrent networks as described recently (Liu et al., 2007). These microcircuits mainly involve intracolumnar synaptic populations in layer II/III (Douglas et al., 1995; Feldmeyer et al., 2002). We found balancing currents, indicated by source mean peak amplitudes, to be higher in supragranular (So1) compared with infragranular (So3) layers.

The finding that cortical silencing balanced these differences points toward a spatial summation of multiple intracortically generated CSD components (Table 3). In whole-cell recordings of thalamocortical-recipient cells, Liu et al. (2007) found a 39\% reduction of excitatory synaptic input. Our results are in line with this study, although a comparison of relative amplitude reductions must bear in mind that CSD amplitudes are not exclusively based on recurrent amplification of afferent input but also reflect later components of intracortical columnar activations that were blocked after drug application (supplemental Note 1, Fig. 1, available at www.jneurosci.org as supplemental material). Hence, the amplitude reduction in thalamocortical-recipient cells reported by Liu et al. (2007) seems to be proportionally less than the reduction of the total CSD amplitude reported here, indicative of integrative intracortical contributions.

\section{Unified functional framework for cortical spectral integration mechanisms.}

From the present data on spatial (laminar) and temporal organization of spectral integration, we propose that information about the spectral energy distribution of sounds is integrated by at least four different input systems (supplemental Fig. 3 available at www.jneurosci.org as supplemental material). In our view, different short- and long-range intracortical projection systems, providing feedforward excitatory or modulatory input, cooperate with afferent thalamocortical input to represent and integrate spectral information in A1 in the following way: afferent thalamocortical connections to layer IV provide broad spectral input with relatively equal amplitudes (Table 2, Fig. 3B) (cf. Bruno and Sakmann, 2006; Liu et al., 2007). Two main and orthogonally oriented intracortical feedforward input systems have been described previously: (1) long-range horizontal projections providing subthreshold spectral information at a cortical site outside the afferent input range (Kadia and Wang, 2003; Metherate et al., 2005) and (2) intracortical recurrent excitation of suprathreshold thalamocortical input (Liu et al., 2007; Wang, 2007). We showed that horizontal inputs also play an important role on a local level (supplemental Fig. 3, short orange and purple arrows, available at www.jneurosci.org as supplemental material). For nearBF stimulation, afferent input converges with horizontal processes on a temporally very precise level (Fig. 6A). This local horizontal input coordinates the activation of adjacent cortical sites in time. Thus, cortical tuning properties result from intracortical connections within (recurrent excitation) and between (horizontal projections) the cortical sites receiving thalamocortical input. These different local processes converging in granular layers act competitively to give rise to sharp cortical tuning properties. In addition, long-range horizontal pathways can provide an effective relay of a broad range of spectral information to adjacent cortical sites terminating in upper layers (sink S2) that are independent from granular inputs.

Finally, we propose a unified functional framework (for schematic illustration, see supplemental Fig. 3, available at www. jneurosci.org as supplemental material) of thalamocortical and local intracolumnar microcircuits defining the response strength of a cortical column. A concurrent network of different long- and short-distant horizontal connections integrates frequency representations of adjacent cortical sites and precisely timed intercolumnar interactions to determine the tuning and response bandwidth of a cortical site.

\section{References}

Ahmed B, Anderson JC, Martin KA, Nelson JC (1997) Map of the synapses onto layer 4 basket cells of the primary visual cortex of the cat. J Comp Neurol 380:230-242.

Andolina IM, Jones HE, Wang W, Sillito AM (2007) Corticothalamic feedback enhances stimulus response precision in the visual system. Proc Natl Acad Sci U S A 104:1685-1690.

Atencio CA, Schreiner CE (2010) Columnar connectivity and laminar processing in cat primary auditory cortex. PLoS ONE 5:e9521.

Bandyopadhyay S, Shamma SA, O'Kanold PO (2010) Dichotomy of functional organization in the mouse auditory cortex. Nat Neurosci 13:361-368.

Bannister AP (2005) Inter- and intra-laminar connections of pyramidal cells in the neocortex. Neurosci Res 53:95-103.

Barth DS, Di S (1990) Three-dimensional analysis of auditory-evoked potentials in rat neocortex. J Neurophysiol 64:1527-1536.

Bitterman Y, Mukamel R, Malach R, Fried I, Nelken I (2008) Ultra-fine frequency tuning revealed in single neurons of human auditory cortex. Nature 451:197-201.

Bruno RM, Sakmann B (2006) Cortex is driven by weak but synchronously active thalamocortical synapses. Science 312:1622-1627.

Budinger E, Heil P, Scheich H (2000a) Functional organization of auditory cortex in the Mongolian gerbil (Meriones unguiculatus). IV. Connections with anatomically characterized subcortical structures. Eur J Neurosci 12:2452-2474.

Budinger E, Heil P, Scheich H (2000b) Functional organization of auditory cortex in the Mongolian gerbil (Meriones unguiculatus). III. Anatomical subdivisions and corticocortical connections. Eur J Neurosci 12:2425-2451.

Chen CM, Lakatos P, Shah AS, Mehta AD, Givre SJ, Javitt DC, Schroeder CE (2007) Functional anatomy and interaction of fast and slow visual pathways in macaque monkeys. Cereb Cortex 17:1561-1569.

Creutzfeldt OD (1983) Cortex cerebri. Heidelberg: Springer.

Cruikshank SJ, Lewis TJ, Connors BW (2007) Synaptic basis for intense thalamocortical activation of feedforward inhibitory cells in neocortex. Nat Neurosci 10:462-468.

Dantzker JL, Callaway EM (2000) Laminar sources of synaptic input to cortical inhibitory interneurons and pyramidal neurons. Nat Neurosci 3:701-707.

Douglas RJ, Martin KA (2007) Recurrent neuronal circuits in the neocortex. Curr Biol 17:R496-R500.

Douglas RJ, Koch C, Mahowald M, Martin KA, Suarez HH (1995) Recurrent excitation in neocortical circuits. Science 269:981-985.

Edeline JM (2003) The thalamo-cortical auditory receptive fields: regulation by the states of vigilance, learning and the neuromodulatory systems. Exp Brain Res 153:554-572.

Edeline JM, Hars B, Hennevin E, Cotillon N (2002) Muscimol diffusion after intracerebral microinjections: a reevaluation based on electrophysiological and autoradiographic quantifications. Neurobiol Learn Mem 78:100-124.

Feldmeyer D, Lübke J, Silver RA, Sakmann B (2002) Synaptic connections between layer 4 spiny neurone-layer $2 / 3$ pyramidal cell pairs in juvenile rat barrel cortex: Physiology and anatomy of interlaminar signalling within a cortical column. J Physiol 538:803-822.

Fox K, Wright N, Wallace H, Glazewski S (2003) The origin of cortical surround receptive fields studied in the barrel cortex. J Neurosci 23:83808391.

Gabernet L, Jadhav SP, Feldman DE, Carandini M, Scanziani M (2005) Somatosensory integration controlled by dynamic thalamocortical feedforward inhibition. Neuron 48:315-327. 
Givre SJ, Schroeder CE, Arezzo JC (1994) Contribution of extrastriate area V4 to the surface-recorded flash VEP in the awake macaque. Vis Res 34:415-428.

Goldschmidt J, Wanger T, Engelhorn A, Friedrich H, Happel M, Ilango A, Engelmann M, Stuermer IW, Ohl FW, Scheich H (2010) Highresolution mapping of neuronal activity using the lipophilic thallium compound TIDDC. I. Protocol and validation of the method. Neuroimage 49:303-315.

Hackett TA (2010) Information flow in the auditory cortical network. Hear Res. Advance online publication. Retrieved Jan. 29, 2010. doi: 10.1016/ j.hearres.2010.01.011.

Harding GW (1992) The currents that flow in the somatosensory cortex during the direct cortical response. Exp Brain Res 90:29-39.

Hirsch JA, Martinez LM (2006) Laminar processing in the visual cortical column. Curr Opin Neurobiol 16:377-384.

Huang CL, Winer JA (2000) Auditory thalamocortical projections in the cat: laminar and area pattern of input. J Comp Neurol 427:302-331.

Imaizumi K, Schreiner CE (2007) Spatial Interactions between spectral integration and frequency gradient in primary auditory cortex. J Neurophysiol 98:2933-2942.

Jellema T, Weijnen JA (1991) A slim needle-shaped multiwire microelectrode for intracerebral recording. J Neurosci Methods 40:203-209.

Kadia SC, Wang X (2003) Spectral integration in A1 of awake primates: Neurons with single- and multipeaked tuning characteristics. J Neurophysiol 89:1603-1622.

Kaur S, Lazar R, Metherate R (2004) Intracortical pathways determine breadth of subthreshold frequency receptive fields in primary auditory cortex. J Neurophysiol 91:2551-2567.

Kaur S, Rose HJ, Lazar R, Liang K, Metherate R (2005) Spectral integration in primary auditory cortex: laminar processing of afferent input, in vivo and in vitro. Neuroscience 134:1033-1045.

Krupa DJ, Ghazanfar AA, Nicolelis MA (1999) Immediate thalamic sensory plasticity depends on corticothalamic feedback. Proc Natl Acad Sci U S A 96:8200-8205.

Kurt S, Deutscher A, Crook JM, Ohl FW, Budinger E, Moeller CK, Scheich H, Schulze H (2008) Auditory cortical contrast enhancing by global winner-take-all inhibitory interactions. PLoS ONE 3:e1735.

Lakatos P, Shah AS, Knuth KH, Ulbert I, Karmos G, Schroeder CE (2005) An oscillatory hierarchy controlling neuronal excitability and stimulus processing in the auditory cortex. J Neurophysiol 94:1904-1911.

Lakatos P, Chen CM, O'Connell MN, Mills A, Schroeder CE (2007) Neuronal oscillations and multisensory interaction in primary auditory cortex. Neuron 53:279-292.

Lee CC, Winer JA (2005) Principles governing auditory cortex connections. Cereb Cortex 15:1804-1814.

Li L, Ebner FF (2006) Balancing bilateral sensory activity: callosal processing modulates sensory transmission through the contralateral thalamus by altering the response threshold. Exp Brain Res 172:397-415.

Linden JF, Schreiner CE (2003) Columnar transformation in auditory cortex? A comparison to visual and somatosensory cortices? Cereb Cortex 13:83-89.

Liu BH, Wu GK, Arbuckle R, Tao HW, Zhang LI (2007) Defining cortical frequency tuning with recurrent excitatory circuitry. Nat Neurosci 10:1594-1600.

Logothetis NK (2008) What we can do and what we cannot do with fMRI. Nature 453:869-878.

Logothetis NK, Kayser C, Oeltermann A (2007) In vivo measurement of cortical impedance spectrum in monkeys: implications for signal propagation. Neuron 55:809-823.

Luhmann HJ, Greuel JM, Singer W (1990) Horizontal interactions in cat striate cortex. II. A current source-density analysis. Eur J Neurosci 2:358-368.

Martin JH, Ghez C (1999) Pharmacological inactivation in the analysis of the central control of movement. J Neurosci Methods 86:145-159.

Matsubara JA, Phillips DP (1988) Intracortical connections and their physiological correlates in the primary auditory cortex (AI) of the cat. J Comp Neurol 268:38-48.

McCormick DA, Shu YS, Hasenstaub A (2003) Balanced recurrent excitation and inhibition in local cortical networks. In: Excitatory-inhibitory balance: synapses, circuits, systems (Hensch T, ed). New York: Kluwer Academic.
Merzenich MM, Knight PL, Roth GL (1975) Representation of cochlea within primary auditory cortex in the cat. J Neurophysiol 38:231-249.

Metherate R, Kaur S, Kawai H, Lazar R, Liang K, Rose HJ (2005) Spectral integration in auditory cortex: mechanisms and modulation. Hear Res 206:146-158.

Miller KD, Pinto DJ, Simons DJ (2001) Processing in layer 4 of the neocortical circuit: new insights from visual and somatosensory cortex. Curr Opin Neurobiol 11:488-497.

Miller LM, Escabí MA, Read HL, Schreiner CE (2001a) Functional convergence of response properties in the auditory thalamocortical system. Neuron 32:151-160.

Miller LM, Escabí MA, Schreiner CE (2001b) Feature selectivity and interneuronal cooperation in the thalamocortical system. J Neurosci 21:8136-8144.

Mitzdorf U (1985) Current source-density method and application in cat cerebral cortex: investigation of evoked potentials and EEG phenomena. Physiol Rev 65:37-100.

Mitzdorf U (1986) The physiological causes of VEP: current source density analysis of electrically and visually evoked potentials. In: Evoked potentials (Cracco R, Bodis-Wollner I, eds), pp 141-154. New York: Liss.

Moeller CK, Kurt S, Happel MFK, Schulze H (2010) Long-range effects of GABAergic inhibition in gerbil primary auditory cortex. Eur J Neurosci 31:49-59.

Nicholson C, Freeman JA (1975) Theory of current source-density analysis and determination of conductivity tensor for anuran cerebellum. J Neurophysiol 38:356-368.

Ohl FW, Scheich H (1997) Orderly cortical representation of vowels based on formant interaction. Proc Natl Acad Sci U S A 94:9440-9444.

Ohl FW, Scheich H, Freeman WJ (2000a) Topographic analysis of epidural pure-tone-evoked potentials in gerbil auditory cortex. J Neurophysiol 83:3123-3132.

Ohl FW, Schulze H, Scheich H, Freeman WJ (2000b) Spatial representation of frequency-modulated tones in gerbil auditory cortex revealed by epidural electrocorticography. J Physiol Paris 94:549-554.

Ojima H, Honda CN, Jones EG (1991) Patterns of axonal collateralization of identified supragranular pyramidal neurons in the cat auditory cortex. Cereb Cortex 1:80-94.

Ojima H, Honda CN, Jones EG (1992) Characteristics of intracellularly injected infragranular pyramidal neurons in cat primary auditory cortex. Cereb Cortex 2:197-216.

Pitts W (1952) Investigations on synaptic transmission. In: CyberneticsTransactions of the Ninth Conference of the Josiah Macy Foundation, New York, pp. 159-166.

Priebe NJ, Ferster D (2008) Inhibition, spike threshold, and stimulus selectivity in primary visual cortex. Neuron 57:482-497.

Razak KA, Fuzessery ZM (2006) Neural mechanisms underlying selectivity for the rate and direction of frequency-modulated sweeps in the auditory cortex of the pallid bat. J Neurophysiol 96:1303-1319.

Razak KA, Fuzessery ZM (2008) Facilitatory mechanisms underlying selectivity for the direction and rate of frequency modulated sweeps in the auditory cortex. J Neurosci 28:9806-9816.

Read HL, Winer JA, Schreiner CE (2001) Modular organization of intrinsic connections associated with spectral tuning in cat auditory cortex. Proc Natl Acad Sci U S A 98:8042-8047.

Rothschild G, Nelken I, Mizrahi A (2010) Functional organization and population dynamics in the mouse primary auditory cortex. Nat Neurosci 13:353-360.

Sakata S, Harris KD (2009) Laminar structure of spontaneous and sensoryevoked population activity in auditory cortex. Neuron 64:404-418.

Schreiner CE, Winer JA (2007) Auditory cortex mapmaking: principles, projections, and plasticity. Neuron 56:356-365.

Schreiner CE, Read HL, Sutter ML (2000) Modular organization of frequency integration in primary auditory cortex. Annu Rev Neurosci 23:501-529.

Schroeder CE, Mehta AD, Givre SJ (1998) A spatiotemporal profile of visual system activation revealed by current source density analysis in the awake macaque. Cereb Cortex 8:575-592.

Schroeder CE, Smiley J, Fu KG, McGinnis T, O’Connell MN, Hackett TA (2003) Anatomical mechanisms and functional implications of multisensory convergence in early cortical processing. Int J Psychophysiol 50:5-17.

Schulze H, Ohl FW, Heil P, Scheich H (1997) Field-specific responses in the 
auditory cortex of the unanaesthetized Mongolian gerbil to tones and slow frequency modulations. J Comp Physiol A 181:573-589.

Steinschneider M, Tenke CE, Schroeder CE, Javitt DC, Simpson GV, Arezzo JC, Vaughan HG Jr (1992) Cellular generators of the cortical auditory evoked potential initial component. Electroencephalogr Clin Neurophysiol 84:196-200.

Steinschneider M, Reser DH, Fishman YI, Schroeder CE, Arezzo JC (1998) Click train encoding in primary auditory cortex of the awake monkey: evidence for two mechanisms subserving pitch perception. J Acoust Soc Am 104:2935-2955.

Stoelzel CR, Bereshpolova Y, Gusev AG, Swadlow HA (2008) The impact of an lgnd impulse on the awake visual cortex: synaptic dynamics and the sustained/transient distinction. J Neurosci 28:5018-5028.

Sutter ML, Schreiner CE, McLean M, O’Connor KN, Loftus WC (1999) Organization of inhibitory frequency receptive fields in cat primary auditory cortex. J Neurophysiol 82:2358-2371.

Swadlow HA (2002) Thalamocortical control of feed-forward inhibition in awake somatosensory "barrel” cortex. Philos Trans R Soc Lond B Biol Sci 357:1717-1727.

Swadlow HA, Gusev AG, Bezdudnaya T (2002) Activation of a cortical column by a thalamocortical impulse. J Neurosci 22:7766-7773.

Szymanski FD, Garcia-Lazaro JA, Schnupp JW (2009) Current source density profiles of stimulus-specific adaptation in rat auditory cortex. J Neurophysiol 102:1483-1490.

Talwar SK, Musial PG, Gerstein GL (2001) Role of mammalian auditory cortex in the perception of elementary sound properties. J Neurophysiol 85:2350-2358.

Tan AY, Zhang LI, Merzenich MM, Schreiner CE (2004) Tone-evoked excitatory and inhibitory synaptic conductances of primary auditory cortex neurons. J Neurophysiol 92:630-643.

Tenke CE, Schroeder CE, Arezzo JC, Vaughan HG Jr (1993) Interpretation of high-resolution current source density profiles: a simulation of sublaminar contributions to the visual evoked potential. Exp Brain Res 94:183-192.
Thomas H, Tillein J, Heil P, Scheich H (1993) Functional organization of auditory cortex in the mongolian gerbil (Meriones unguiculatus). I. Electrophysiological mapping of frequency representation and distinction of fields. Eur J Neurosci 5:882-897.

Thomson AM, Bannister AP (2003) Interlaminar connections in the neocortex. Cereb Cortex 13:5-14.

Tomioka R, Okamoto K, Furuta T, Fujiyama F, Iwasato T, Yanagawa Y, Obata K, Kaneko T, Tamamaki N (2005) Demonstration of long-range GABAergic connections distributed throughout the mouse neocortex. Eur J Neurosci 21:1587-1600.

Velenovsky DS, Cetas JS, Price RO, Sinex DG, McMullen NT (2003) Functional subregions in primary auditory cortex defined by thalamocortical terminal arbors: an electrophysiological and anterograde labeling study. J Neurosci 23:308-316.

Wallace MN, Kitzes LM, Jones EG (1991) Intrinsic inter- and intralaminar connections and their relationship to the tonotopic map in cat primary auditory cortex. Exp Brain Res 86:527-544.

Wang X (2007) A sharper view from the top. Nat Neurosci 10:1509-1511.

Wehr M, Zador AM (2003) Balanced inhibition underlies tuning and sharpens spike timing in auditory cortex. Nature 426:442-446.

Winer JA, Miller LM, Lee CC, Schreiner CE (2005) Auditory thalamocortical transformation: structure and function. Trends Neurosci 28:255-263.

Wu Y, Yan J (2007) Modulation of the receptice fields of midbrain neurons elicited by thalamic electrical stimulation through corticofugal feedback. J Neurosci 40:10651-10658.

Wu GK, Arbuckle R, Liu BH, Tao HW, Zhang LI (2008) Lateral sharpening of cortical frequency tuning by approximately balanced inhibition. Neuron 58:132-143.

Yamauchi T, Hori T, Takahashi T (2000) Presynaptic inhibition by Muscimol through $\mathrm{GABA}_{\mathrm{B}}$ receptors. Eur J Neurosci 12:3433-3436.

Zandieh S, Hopf R, Redl H, Schlag MG (2003) The effect of ketamine/xylazine anesthesia on sensory and motor evoked potentials in the rat. Spinal Cord 41:16-22. 